\title{
WEAKLY ALMOST PERIODIC FUNCTIONS AND ALMOST CONVERGENT FUNCTIONS ON A GROUP
}

\author{
BY \\ CHING CHOU(1)
}

\begin{abstract}
Let $G$ be a locally compact group, $U C(G)$ the space of bounded uniformly continuous complex functions on $G, C_{0}(G)$ the subspace of $U C(G)$ consisting of functions vanishing at infinity. Let $W(G)$ be the space of weakly almost periodic functions on $G$ and $W_{0}(G)$ the space of functions in $W(G)$ such that their absolute values have zero invariant mean. If $G$ is amenable let $F(G)$ be the space of almost convergent functions in $U C(G)$ and $F_{0}(G)$ the space of functions in $F(G)$ such that their absolute values are almost convergent to zero. The inclusive relations among the above-mentioned spaces are studied. It is shown that if $G$ is noncompact and satisfies certain conditions, e.g. $G$ is nilpotent, then each of the quotient Banach spaces $U C(G) / W(G), W_{0}(G) / C_{0}(G)$, $F_{0}(G) / W_{0}(G)$ contains a linear isometric copy of $l^{\infty}$. On the other hand, an example of a noncompact group $G$ is given which satisfies the condition that $C_{0}(G)=w_{0}(G)$.
\end{abstract}

1. Introduction. Let $G$ be a locally compact group, $C(G)$ the space of bounded continuous complex-valued functions on $G$ with the sup norm, $U C(G)$ the subspace of $C(G)$ consisting of uniformly continuous functions and $C_{0}(G)$ the subspace of $C(G)$ consisting of functions vanishing at infinity. Let $W(G)$ be the space of all functions in $C(G)$ which are weakly almost periodic (w.a.p.). Denote the unique invariant mean on $W(G)$ by $m$ and set $W_{0}(G)=\{f \in W(G)$ : $m(|f|)=0$ \}. If $G$ is amenable we shall also consider the spaces $F L(G)=$ $\left\{f \in U C(G): \mu(f)=\right.$ a fixed constant $d_{l}(f)$ as $\mu$ runs through the set of all left invariant means on $U C(G)\}$ and $F L_{0}(G)=\{f \in U C(G):|f| \in F L(G)$ and $d_{l}(|f|)=0$. The following inclusive relations are well known: $W(G) \subset U C(G)$, $C_{0}(G) \subset W_{0}(G)$ if $G$ is noncompact; $W(G) \subset F L(G), W_{0}(G) \subset F L_{0}(G)$ if $G$ is amenable, cf. [1]. It is obvious that if $G$ is compact then all of the above inclusive relations can be reversed. We are interested in deciding whether each of the above inclusive relations is proper if $G$ is noncompact.

Burckel [1] proved that $C(G)=W(G)$ if and only if $G$ is compact. In

Presented to the Society, November 7, 1972; received by the editors January 15, 1974. AMS (MOS) subject classifications (1970). Primary 22A10, 43A07, 46N05; Secondary 22D99, 43A60, 46E15.

(1) Supported in part by NSF grant PO34190. 
[9] Granirer provided the following improvement of his result:

(1) $U C(G)=W(G)$ if and only if $G$ is compact.

(2) If $G$ is noncompact and amenable then the quotient Banach space $U C(G) / W(G)$ is nonseparable.

Since $F L(G)$ lies between $U C(G)$ and $W(G)$ it is natural to ask whether $F L(G) \supsetneqq W(G)$ if $G$ is as in (2) above. We shall prove that it is indeed so if $G$ is almost connected as a consequence of the following.

THEOREM. Let $G$ be a o-compact locally compact noncompact amenable group, $f \in U C(G)$. Then $f \cdot F L(G) \subset F L(G)$ if and only if $f \in F L_{0}(G) \oplus \mathbf{C}$. (The constant functions on $G$ are identified with $\mathrm{C}$.)

The discrete version of the above theorem is contained in [4].

Another natural question one may ask is the following: If the condition "amenable" is skipped from (2) above can one still make the same conclusion? To give a partial solution and for further discussions we like to take a digression here.

Definition. A locally compact group $G$ is said to have property (E) if either $G$ is compact or $G$ contains a subset $X$ such that $\mathrm{cl} X$ is noncompact and for each neighborhood $U$ of the identity $e$ of $G, \bigcap\left\{x^{-1} U x: x \in X \cup X^{-1}\right\}$ is again a neighborhood of $e$.

The class of groups with property (E) is quite large, e.g., discrete groups, abelian groups and the group $G L(n, R)$ all have property $(E)$. But there exists a nilpotent group which fails to have property (E). See $\$ 4$ for the details.

A theorem parallel to (2) above is the following.

THEOREM. Let $G$ be a locally compact noncompact group with property (E). Then $U C(G) / W(G)$ contains a linear isometric copy of $l^{\infty}$.

Let $A(G)$ be the set of all almost periodic functions in $C(G)$. Then $W(G)=W_{0}(G) \oplus A(G)$, cf. [1]. Since the properties of almost periodic functions are better known, the mysterious part of $W(G)$ is $W_{0}(G)$. In $[1, \mathrm{p} .74]$ Burckel proved that if $G$ is an abelian locally compact noncompact group then $C_{0}(G) \subsetneq W_{0}(G)$. His proof depends heavily on the fact that $G$ is abelian. He went on to conjecture that the abelian hypothesis is inessential. We shall give a noncompact solvable group $G$ with $C_{0}(G)=W_{0}(G)$. On the other hand we have the following.

THEOREM. Let $G$ be a noncompact locally compact group which either has property (E) or is nilpotent. Then $W_{0}(G) / C_{0}(G)$ contains a linear isometric copy of $l^{\infty}$. 
Let $\cdot F_{0}(G)=\{f \in U C(G): \mu(|f|)=0$ for each left or right invariant mean $\mu$ on $U C(G)$ \}. It is well known that $F_{0}(G) \supset W_{0}(G)$ if $G$ is amenable. In [1, Theorem 3.19] Burckel proved that $F_{0}(\mathbf{R}) \supsetneqq W_{0}(\mathbf{R})$ where $\mathbf{R}$ is the additive group of reals with the usual topology. We shall provide the following generalization.

THEOREM. Let $G$ be a noncompact locally compact group which is either amenable and with noncompact center or is nilpotent or is connected and solvable. Then $F_{0}(G) / W_{0}(G)$ contains a linear isometric copy of $l^{\infty}$.

We do not even know whether the above theorem is true for every discrete amenable group.

2. Preliminaries and notations. Let $G$ be a locally compact group with a fixed left Haar measure $\lambda$ (or $\lambda_{G}$ if there is a chance for confusion). If $f$ is a Borel measurable function on $G$ and $B$ is a Borel subset of $G$, the integral of $f$ on $B$ with respect to $\lambda$ is denoted by $\int_{B} f(x) d x$. The Banach space of all essentially bounded complex-valued Borel functions with the ess sup norm, $\|\cdot\|_{\infty}$, is denoted by $L^{\infty}(G)$. The space of integrable complex functions with respect to $\lambda$ is denoted by $L^{1}(G)$. As is well known, the dual Banach space of $L^{1}(G)$ can be identified with $L^{\infty}(G)$. We shall write $\langle f, g\rangle$ for $\int_{G} f(x) g(x) d x, f \in L^{\infty}(G)$, $g \in L^{1}(G)$.

If $f$ is a function on $G$ and $x \in G, l_{x} f\left\{r_{x} f\right\}$, the left \{right $\}$ translation of $f$ by $x$, is defined by $\left(l_{x} f\right)(y)=f(x y)\left\{\left(r_{x} f\right)(y)=f(y x)\right\}$. A function $f \in C(G)$ is said to be left \{right\} uniformly continuous if, given $\epsilon>0$, there is a neighborhood $U$ of the identity $e$ of $G$ such that $\left\|f-r_{x} f\right\|_{\infty}<\epsilon$ $\left\{\left\|f-l_{x} f\right\|_{\infty}<\epsilon\right\}$ for $x \in U . f$ is said to be uniformly continuous if it is both right and left uniformly continuous. $U C(G)$, the space of uniformly continuous functions in $C(G)$, is a closed subalgebra of $C(G)$ and is closed under translations, i.e., if $f \in U C(G)$ and $x \in G$ then $l_{x} f$ and $r_{x} f$ also belong to $U C(G)$.

$\mu \in U C(G)^{*}$ is called a mean if $\|\mu\|=\mu(1)=1$. (1 also stands for the constant one function on $G$.) A mean is left \{right invariant if $\mu\left(l_{x} f\right)=\mu(f)$ $\left\{\mu\left(r_{x} f\right)=\mu(f)\right\}$ for $x \in G$ and $f \in U C(G)$. The set of left \{right $\}$ [twosided] invariant means on $U C(G)$ is denoted by $M L(G)\{M R(G)\}[M(G)] . G$ is said to be amenable if $M(G)$ is nonempty which is equivalent to the weaker condition that $M L(G)$ is nonempty. It is known that solvable groups and compact groups are amenable. We refer the readers to [5], [10] for the general facts concerning amenable groups and invariant means. It is clear that invariant means can also be defined for other closed linear subspaces of $L^{\infty}(G)$ which are closed under translations. 
If $G$ is amenable then the spaces $F L(G), F L_{0}(G)$ are defined in $\S 1$. If $f \in F L(G)$ then we shall call it a left almost convergent function. Similarly, let $F R(G)=\left\{f \in U C(G): \mu(f)=\right.$ a constant $d_{r}(f)$ as $\mu$ runs through $\left.M R(G)\right\}$ and $F R_{0}(G)=\left\{f \in U C(G):|f| \in F R(G), d_{r}(|f|)=0\right\}$. Clearly $F L_{0}(G) \subset$ $F L(G)$ and $F R_{0}(G) \subset F R(G)$. Let $F(G)=F L(G) \cap F R(G)$ be the space of (two-sided) almost convergent functions in $U C(G)$. Since $M L(G) \cap M R(G)=$ $M(G) \neq \varnothing$ one sees that $F(G)=\{f \in U C(G): \mu(f)=$ a constant $d(f)$ as $\mu$ runs through $M L(G) \cup M R(G)\}$ and $d(f)=d_{l}(f)=d_{r}(f)=d(f)$ if $f \in F(G)$. Set $F_{0}(G)=\{f \in U C(G):|f| \in F(G), d(|f|)=0\}$. All the spaces mentioned in this paragraph are closed linear subspaces of $U C(G)$.

The convolution of two Borel measurable functions $f$ and $g$ on $G$ is defined by $(f * g)(x)=\int_{G} f(t) g\left(t^{-1} x\right) d t$. Note that if $f \in L^{\infty}(G)$ and $\varphi \in L^{1}(G)$ then $\varphi * f$ is bounded and right uniformly continuous and $f * \varphi^{\sim}$ is bounded and left uniformly continuous. ( $\varphi^{\sim}$ is defined by $\varphi^{\sim}(x)=\varphi\left(x^{-1}\right)$.) Note also that $L^{1}(G)$ is a Banach algebra with convolution as multiplication. Let $P(G)=\left\{\varphi \in L^{1}(G):\|\varphi\|_{1}=1, \varphi \geqslant 0\right\}$. We shall need the following wellknown but elementary facts.

LEMMA 2.1. Let $G$ be a locally compact group.

(a) For $f \in L^{\infty}(G), \varphi, \psi \in L^{1}(G)$,

$$
\left\langle f * \psi^{\sim}, \varphi\right\rangle=\langle f, \varphi * \psi\rangle=\left\langle(1 / \Delta) \varphi^{\sim} * f, \psi\right\rangle .
$$

(b) $\left\{\varphi * f * \psi^{\sim}: \varphi, \psi \in P(G), f \in L^{\infty}(G)\right\}$ is dense in $U C(G)$.

Here $\Delta$ is the modular function for the left Haar measure $\lambda$. For a proof of (a), cf. [15, Lemma 3.1].

It is interesting to point out that a mean $\mu \in U C(G)^{*}$ is left \{right invariant if and only if $\mu(\varphi * f)=\mu(f)\left\{\mu\left(f * \varphi^{\sim}\right)=\mu(f)\right\}$ for $\varphi \in P(G)$ and $f \in U C(G)$, cf. [10, p. 27]. We shall also need the following well-known lemma.

LEMMA 2.2. Let $G$ be a locally compact amenable group. Then

$$
\begin{aligned}
F L(G) & =\text { the closed linear span of }\left\{f-l_{x} f: f \in U C(G), x \in G\right\} \cup\{1\} \\
& =\text { the closed linear span of }\{f-\varphi * f: f \in U C(G), \varphi \in P(G)\} \cup\{1\} .
\end{aligned}
$$

For a proof of the above lemma cf. [5, Theorem 9.1] or [15, Theorem 7.3] Of course a similar result holds for $F R(G)$.

If a locally compact amenable group $G$ is also $\sigma$-compact then there exists a sequence of compact neighborhoods $U_{n}$ of the identity $e$ which satisfies the following two conditions (cf. [3]): 


$$
U_{n} \subset U_{n+1}, n=1,2, \cdots ; \quad \bigcup_{n=1}^{\infty} U_{n}=G
$$

and

$$
\lim _{n}\left(\lambda\left(x U_{n} \Delta U_{n}\right) / \lambda\left(U_{n}\right)\right)=0
$$

uniformly on compact subsets of $G$. Here $A \Delta B=(A \backslash B) \cup(B \backslash A)$. As in [3], we shall call such a sequence an $F$-sequence.

Lemma 2.3 (cf. [3]). Let $\left(U_{n}\right)$ be an F-sequence for a locally compact amenable group G. Let $\varphi_{n}=1 / \lambda\left(U_{n}\right) \chi_{U_{n}}$, where $\chi_{U_{n}}$ is the characteristic function of $U_{n}$. Then $f \in U C(G)$ is left almost convergent to a constant $c$ if and only if $(1 / \Delta) \varphi_{n}^{\tilde{n}} * f$ converges to the constant function $c$ in uniform topology.

Weakly almost periodic functions were introduced by Eberlein [8]. Recall that $f \in C(G)$ is w.a.p. if the set $\left\{l_{x} f: x \in G\right\}$ is relatively compact in the weak topology of $C(G)$. It is well known that $W(G)$, the set of all w.a.p. functions in $C(G)$, is a closed subalgebra of $U C(G)$ and it is closed under translations. Furthermore, there is a unique invariant mean $m$ (or $m_{G}$ if there is a chance for confusion) on $W(G)$ no matter whether $G$ is amenable or not. (This is the well-known Ryll-Nardzewski fixed point theorem.) We refer the reader to [1] for a quite complete account of the theory of w.a.p. functions. A useful criterion for $f \in C(G)$ to be w.a.p. is the following: $f$ is w.a.p. if and only if for each sequence of elements in $G$ there is a subsequence $\left(a_{n}\right)$ such that $l_{a_{n}} f$ converges pointwisely on $\beta G$ to a continuous function $g$. Here $\beta G$ is the StoneČech compactification of the locally compact space $G$ and for each $h \in C(G)$ its continuous extension to $\beta G$ is again denoted by $h$. We shall use the above criterion several times in the sequel without any further explanation.

Lemma 2.4 (cf. [6, Lemma 5.4] or [1, Theorem 3.14]). Let $H$ be an open subgroup of a locally compact group $G, f \in W(H)$. Define $f^{\prime} \in C(G)$ by setting $f^{\prime}=f$ on $H$ and $f^{\prime}=0$ on $G \backslash H$. Then $f^{\prime} \in W(G)$.

In [1], $H$ is assumed to be a normal subgroup. But a careful examination of his proof tells us that "normality" is not required.

If $G$ is a locally compact group, $A(G)$ will denote the group of bicontinuous automorphisms of $G$. We shall give $A(G)$ the compact open topology. If $K$ and $H$ are locally compact groups and $\eta: K \rightarrow A(H)$ is a continuous homomorphism, let $G=\{(x, y): x \in H, y \in K\}$ be the group with product topology and with multiplication given by $(x, y)\left(x^{\prime}, y^{\prime}\right)=\left(x \cdot \eta(y) x^{\prime}, y y^{\prime}\right)$. Then $G$ is called a topological semidirect product of $H$ and $K$. $G$ will be denoted by $H \times \times_{\eta} K$. 
3. Multipliers for $F L(G) . f \in U C(G)$ is called a multiplier for $F L(G)$ if $f \cdot F L(G) \subset F L(G)$. We have the following characterization for the multipliers.

THEOREM 3.1. Let $G$ be a noncompact $\sigma$-compact locally compact amenable group, $f \in U C(G)$. Then $f \cdot F L(G) \subset F L(G)$ if and only if $f \in \mathrm{C} \oplus F L_{0}(G)$.

Proof. The "if" part is easy.(2) Let $\left(U_{n}\right)$ be an $F$-sequence for $G$ and let $\varphi_{n}=\left(1 / \lambda\left(U_{n}\right)\right) \chi_{U_{n}}$. Assume that $f$ is a multiplier for $F L(G)$. Fix $\xi$, $\eta \in P(G), x \in G$ and set

$$
\psi_{n}=(1 / \Delta) \xi^{\sim} *\left(f \cdot \varphi_{n}-l_{x}\left(f \cdot \varphi_{n}\right)\right) * \eta .
$$

Note that $\left(\psi_{n}\right)$ is a sequence in $L^{1}(G)$. Let $h \in L^{\infty}(G)$ and set $g=$ $\xi * h * \eta \sim$.

$$
\begin{aligned}
\left\langle h, \psi_{n}\right\rangle & =\left\langle\xi * h * \eta \sim, f \cdot \varphi_{n}-l_{x}\left(f \cdot \varphi_{n}\right)\right\rangle \quad \text { (by Lemma 2.1(a)) } \\
& =\left\langle g, f \cdot \varphi_{n}\right\rangle-\left\langle l_{x-1} g, f \cdot \varphi_{n}\right\rangle \\
& =\left\langle g-l_{x^{-1}} g, f \cdot \varphi_{n}\right\rangle=\left\langle f \cdot\left(g-l_{x^{-1}} g\right), \varphi_{n}\right\rangle .
\end{aligned}
$$

Since $g-l_{x^{-1}} g \in F L(G)$, by assumption $f \cdot\left(g-l_{x^{-1}} g\right) \in F L(G)$. By Lemma 2.3,

$$
\begin{aligned}
\lim _{n}\left\langle h, \psi_{n}\right\rangle & =\lim _{n}\left\langle f \cdot\left(g-l_{x^{-1}} g\right), \varphi_{n}\right\rangle \\
& =\lim _{n}\left((1 / \Delta) \varphi_{n}^{\sim} *\left(f \cdot\left(g-l_{x^{-1}} g\right)\right)\right)(e) \\
& =d_{l}\left(f \cdot\left(g-l_{x^{-1}} g\right)\right) .
\end{aligned}
$$

(1) implies that the sequence $\left(\psi_{n}\right)$ is weakly Cauchy in $L^{1}(G)$. But $L^{1}(G)$ is weakly sequentially complete (cf. [7, p. 374]), therefore, there exists $\psi \in L^{1}(G)$ such that

$$
\lim _{n}\left\langle h, \psi_{n}\right\rangle=\langle h, \psi\rangle, \quad h \in L^{\infty}(G) .
$$

We claim that $\psi=0$ almost everywhere. Note first that since $G$ is noncompact, $\lambda\left(U_{n}\right) \rightarrow \infty$ as $n \rightarrow \infty$, and hence

$$
\left\|f \cdot \varphi_{n}-l_{x}\left(f \cdot \varphi_{n}\right)\right\|_{\infty} \leqslant 2\left(1 / \lambda\left(U_{n}\right)\right)\|f\|_{\infty} \rightarrow 0 .
$$

Therefore, $\lim _{n}\left\|\psi_{n}\right\|_{\infty}=0$. If $\psi \neq 0$ almost everywhere, then there exist $\delta>0$ and a compact set $K$ such that $\lambda(K)>0$ and $|\psi| \geqslant \delta$ on $K$. Set $h(x)=$ $(1 / \psi(x))$ if $x \in K$ and $=0$ otherwise. Then $h \in L^{\infty}(G)$, and

(2) Let $f=d_{l}(f)+f_{0} \in \mathrm{C} \oplus F L_{0}(G)$. If $g \in F L(G)$ and $\mu \in M L(G)$ then $\left|\mu\left(f_{0} g\right)\right|<\mu\left(\left|f_{0} g\right|\right)<\|g\|_{\infty} \mu\left(\left|f_{0}\right|\right)=0$. Therefore $\mu(f \cdot g)=d_{l}(f) \mu(g)=d_{l}(f) d_{l}(g)$ and hence $f \cdot g \in F L(G)$. 


$$
\left|\left\langle h, \psi_{n}\right\rangle\right| \leqslant(1 / \delta) \lambda(K)\left\|\psi_{n}\right\|_{\infty} \rightarrow 0 .
$$

On the other hand, $\langle h, \psi\rangle=\lambda(K)>0$. This contradicts (2). Therefore $\psi=0$ almost everywhere as we claimed. Combining this fact with (1) and (2) we get

$$
d_{l}\left(f \cdot\left(g-l_{x^{-1}} g\right)\right)=0,
$$

if $g \in E=\left\{\xi * h * \eta^{\sim}: \xi, \eta \in P(G), h \in L^{\infty}(G)\right\}$. Since $E$ is uniformly dense in $U C(G)$ (Lemma 2.1(b)), since the linear span of $\left\{k-l_{x} k: k \in U C(G)\right\}$ $\cup\{1\}$ is uniformly dense in $F L(G)$ (Lemma 2.2) and since $d_{l}(f \cdot c)=c d_{l}(f)$, $c$ the constant $c$ function on $G$, we derive from (3) that

$$
d_{l}(f \cdot g)=d_{l}(f) d_{l}(g) \text { if } g \in F L(G)
$$

Note that it is easy to see that if $g \in F L(G)$ then $\bar{g}$, the conjugate of $g$, also belongs to $F L(G)$ and $d_{l}(\bar{g})=\overline{d_{l}(g)}$. By (4), we have

$$
\begin{aligned}
d_{l}\left(\left|f-d_{l}(f)\right|^{2}\right) & =d_{l}(f \cdot \bar{f})-d_{l}(\bar{f}) d_{l}(f)-\overline{d_{l}(f)} d_{l}(f)+d_{l}(f) \overline{d_{l}(f)} \\
& =0 .
\end{aligned}
$$

Since $U C(G)$ is a commutative $C^{*}$-algebra it is isomorphic to $C(X)$ for some compact set $X$. In particular, each $\mu \in M L(G)$ can be considered as a measure on $X$. Therefore $\mu\left(\left|f-d_{l}(f)\right|\right)=0$ for $\mu \in M L(G)$, i.e., $f-d_{l}(f)$ $\in F L_{0}(G)$ and the proof is completed.

Our method of proof is a generalization of the discrete version of the above theorem given in [4].

Recall that $A(G)$ denotes the algebra of almost periodic functions in $C(G)$. It is well known that if $f \in A(G)$ and $f \neq 0$ then $m(|f|)>0$, cf. [11, p. 250]. In particular, if $f \in A(G)$ is not a constant function then $f \notin F L_{0}(G) \oplus \mathrm{C}$.

COROllary 3.2. If $G$ is as in Theorem 3.1 and $A(G)$ contains a nonconstant function then $F L(G)$ is not closed under multiplication and hence $U C(G) \underset{\supsetneqq}{\supset} L(G) \supsetneqq W(G)$.

$A(G) \neq \mathrm{C}$ if and only if $G$ has a nontrivial finite dimensional unitary representation. Therefore $A(G) \neq \mathrm{C}$ if either $G$ is solvable or is almost connected and amenable. Note that if $\boldsymbol{G}$ is almost connected and amenable then $\boldsymbol{G}$ is a topological group extension of a solvable group by a compact group, cf. [10, p. 53]. On the other hand there exist discrete amenable groups with no nontrivial finite dimensional unitary representations. For example, the locally finite group $G=\bigcup_{n=1}^{\infty} A_{n}$, where $A_{n}$ is the group of even permutations on $\{1,2, \cdots, n\}$, satisfies $A(G)=\mathrm{C}$. 
We like to conjecture that if $G$ is noncompact then $F L(G) \underset{\supsetneqq}{\supset} L_{0}(G) \oplus \mathrm{C}$ no matter whether $A(G)=\mathrm{C}$ or not. It is known that if $G$ is noncompact and if $F$ is the set of all left almost convergent functions on $L U C(G)$ then $F$ is not an algebra.(3) Therefore our conjecture is also true for infinite discrete amenable groups.

REMARK. Let $E=\{f \in U C(G): \mu(f)=$ a constant as $\mu$ runs through $M(G)$ ). Then $E \supset F L(G)$. In $[9$, p. 62], E. Granirer proved that $U C(G) / E$ is nonseparable if $G$ is a noncompact locally compact amenable group by applying a deep theorem of his [9, Theorem 5]. It is also a consequence of the following result of ours [3, Theorem 5.3]: If $G$ is a $\sigma$-compact locally compact noncompact amenable group then $M(G)$ contains at least $2^{c}$ elements. Indeed, as mentioned in [9] to see that $U C(G) / E$ is nonseparable we may assume that $G$ is $\sigma$-compact. If $U C(G) / E$ is separable then there exists a countable set $\left(f_{n}\right)$ in $U C(G)$ such that $E+$ the linear span of $\left(f_{n}\right)$ is dense in $U C(G)$. Therefore each $\mu \in M(G)$ is dciermined by $\left(\mu\left(f_{n}\right)\right)$. Hence $M(G)$ contains at most $c$ elements which contradicts the result in [3] quoted above.

We are unable to prove the analogy of Theorem 3.2 for $F(G)$. Namely, we do not know whether $f \cdot F(G) \subset F(G)$ implies that $f \in F_{0}(G) \oplus$ C. But we do have the following weaker result.

THEOREM 3.3. Let $G$ be a noncompact o-compact locally compact amenable group with $A(G) \neq \mathrm{C}$. Then $F(G)$ is not closed under multiplication and hence $F(G) \supsetneqq W(G)$.

Proof. Suppose that $F(G)$ is closed under multiplication. Let $f \in F(G)$. Let $\xi, \eta, \alpha \in P(G)$ and $x \in G$ be fixed. Consider the sequence $\left(\psi_{n}\right)$ in $L^{1}(G)$ where

$$
\psi_{n}=(1 / \Delta) \xi^{\sim} *\left(\left(f \cdot \varphi_{n}-l_{x}\left(f \cdot \varphi_{n}\right)\right)-\left(f \cdot \varphi_{n}-l_{x}\left(f \cdot \varphi_{n}\right)\right) * \alpha\right) * \eta .
$$

Let $h \in L^{\infty}(G)$ and set $h^{\prime}=\xi * h * \eta^{\sim}-\xi * h * \eta^{\sim} * \alpha^{\sim}$. Note that

$$
\begin{aligned}
\left\langle h, \psi_{n}\right\rangle & =\left\langle\xi * h * \eta^{\sim},\left(f \cdot \varphi_{n}-l_{x}\left(f \cdot \varphi_{n}\right)\right)-\left(f \cdot \varphi_{n}-l_{x}\left(f \cdot \varphi_{n}\right)\right) * \alpha\right\rangle \\
& =\left\langle\xi * h * \eta^{\sim}-\xi * h * \eta^{\sim} * \alpha^{\sim}, f \cdot \varphi_{n}-l_{x}\left(f \cdot \varphi_{n}\right)\right\rangle \\
& =\left\langle h^{\prime}-l_{x^{-1}} h^{\prime}, f \cdot \varphi_{n}\right\rangle=\left\langle f \cdot\left(h^{\prime}-l_{x^{-1}} h^{\prime}\right), \varphi_{n}\right\rangle .
\end{aligned}
$$

By Lemma $2.2, h^{\prime} \in F R(G)$ and $h^{\prime}-l_{x-1} h^{\prime} \in F L(G)$. We claim that $h^{\prime}-$ $l_{x-1} h^{\prime} \in F R(G)$, and hence belongs to $F(G)$. Indeed, since $d_{r}\left(h^{\prime}\right)=0$, by Lemma 2.2, $h^{\prime}$ and hence $l_{x} h^{\prime}$ belong to the closed linear span of $\left\{g-r_{y} g\right.$ :

(3) We wish to thank Professor E. Granirer for communicating this result to us. 
$g \in U C(G), y \in G\}$. Therefore $h^{\prime}-l_{x^{-1}} h^{\prime} \in F R(G)$ as we claimed. By assumption $f \cdot\left(h^{\prime}-l_{x^{-1}} h^{\prime}\right) \in F(G)$, and hence, by (1) and Lemma 2.3,

$$
\lim _{n}\left\langle f \cdot\left(h^{\prime}-l_{x^{-1}} h^{\prime}\right), \varphi_{n}\right\rangle=d\left(f \cdot\left(h^{\prime}-l_{x^{-1}} h^{\prime}\right)\right) .
$$

As in the proof of Theorem 3.1, one sees that $\lim _{n}\left\|\psi_{n}\right\|_{\infty}=0$ and that if $J=$ $\left\{\xi * h * \eta^{\sim}-\xi * h * \eta^{\sim} * \alpha^{\sim}: h \in L^{\infty}(G), \xi, \eta, \alpha \in P(G)\right\}$ then

$$
d\left(f \cdot\left(h^{\prime}-l_{x} h^{\prime}\right)\right)=0 \quad \text { if } x \in G \text { and } h^{\prime} \in J .
$$

Note that the linear span of $J \cup\{1\}$ is dense in $F R(G)$ since the linear span of $\left\{g-g * \alpha^{\sim}: g \in U C(G), \alpha \in P(G)\right\} \cup\{1\}$ is dense in $F R(G)$ (Lemma 2.2) and $\left\{\xi * h * \eta^{\sim}: \xi, \eta \in P(G), h \in L^{\infty}(G)\right\}$ is dense in $U C(G)$ (Lemma 2.1(b)). By (2), $d\left(f \cdot\left(g-l_{x} g\right)\right)=0$ if $g \in F R(G), f \in F(G)$. In particular, $d\left(\left|g-l_{x} g\right|^{2}\right)$ $=0$ if $g \in F R(G), x \in G$. Let $g$ be a nonconstant function in $A(G)$. (Then $g \in F(G)$.) Then for at least one $x, d\left(\left|g-l_{x} g\right|^{2}\right) \neq 0$. This is a contradiction.

4. $C_{0}(G)$ and $W_{0}(G)$. It is well known that if $G$ is a noncompact locally compact group then $C_{0}(G) \subset W_{0}(G)$, cf. [1]. In [1, Theorem 4.17], Burckel proved that if $G$ is further assumed to be abelian then the inclusion is proper, i.e., $C_{0}(G) \underset{f}{\subsetneq} W_{0}(G)$. He conjectured that the abelian hypothesis is inessential. In this section we shall prove that his theorem is true for a much bigger class of groups; and, on the other hand, there does exist a noncompact group $G$ with $C_{0}(G)=W_{0}(G)$.

We shall start with some simple observations.

LEMMA 4.1. Let $G$ and $H$ be locally compact groups.

(1) If $\varphi$ is a continuous homomorphism from $G$ onto $H$ and if $C_{0}(H)$

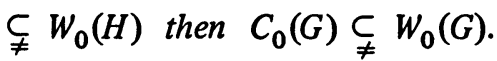

(2) If $\varphi$ is a continuous homomorphism of $G$ onto $H$ and if both $H$ and $\operatorname{ker} \varphi$ are noncompact then $C_{0}(G) \underset{\ddagger}{\subsetneq} W_{0}(G)$.

(3) If $H$ is a noncompact open subgroup of $G$ and $G / H$ is infinite then $C_{0}(G) \underset{f}{\subsetneq} W_{0}(G)$.

Proof. Let $\varphi$ be a continuous homomorphism from $G$ onto $H$ and $f \in W(H)$. Then (a) $f \circ \varphi \in W(G)$, (b) $m_{G}(f \circ \varphi)=m_{H}(f)$. (a) is [1, Theorem 1.8]. To see (b), note that if $m_{H}(f)=c$ then $c \in$ closed convex hull of $\left\{l_{y} f: y \in H\right\}$, cf. [1, Theorem 1.25]. Therefore, $c \in$ closed convex hull of $\left\{l_{x}(f \circ \varphi): x \in G\right\}$. Then $m_{G}(f \circ \varphi)=c$, cf. [1, Theorem 1.25].

Now for (1), choose $f \in W_{0}(H) \backslash C_{0}(H)$. Then $f \circ \varphi \in W_{0}(G) \backslash C_{0}(G)$. For (2), choose $f \in C_{0}(H), f \neq 0$. Then $f \in W_{0}(H)$ and $f \circ \varphi \in W_{0}(G) \backslash C_{0}(G)$. 
(3) follows from Lemma 2.4 by taking $f \equiv 1$ on $H$ and $\equiv 0$ on $G \backslash H$.

COROLlARY 4.2. If a locally compact group $G$ is either nonunimodular

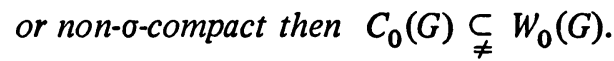

Proof. If $G$ is nonunimodular then the modular function $\Delta$ is a continuous homomorphism of $G$ onto a noncompact closed subgroup of the multiplicative group of positive reals. Therefore $C_{0}(G) \underset{\neq}{\subsetneq} W_{0}(G)$ by Lemma 4.1(1) and Burckel [1, Theorem 4.17].

If $G$ is not $\sigma$-compact, choose a noncompact $\sigma$-compact open subgroup $H$ of $G$, cf. $\left[9\right.$, p. 64]. Clearly $G / H$ is infinite. Therefore $C_{0}(G) \underset{\ddagger}{\subsetneq} W_{0}(G)$ by Lemma 4.1(3).

Definition 4.3. Let $G$ be a locally compact group. A set $X \subset G$ is said to be an $E$-set if given a neighborhood $U$ of $e$ the set $\bigcap\left\{x U x^{-1}: x \in\right.$ $\left.X \cup X^{-1}\right\}$ is again a neighborhood of $e$.

Note that if the right and left uniform structures of $G$ are equivalent then each subset of $G$ is an $E$-set. Note also that each compact subset of a locally compact group $G$ is an $E$-set. Recall that a locally compact group $G$ is said to have property (E) if either $G$ is compact or $G$ contains an $E$-set which is not relatively compact (cf. $\S 1$ ). While we cannot characterize the class of groups with property $(E)$ we do have a large number of examples.

Proposition 4.4. The following locally compact groups have property (E): (1) a group with equivalent right and left uniform structures, e.g., an abelian group, a discrete group, (2) a group with noncompact center, e.g., $G L(n, \mathbf{R})$, or the subgroup of $G L(n, \mathbf{R})$ consisting of upper-triangular metrics $\left(x_{i j}\right)$ with $x_{i i}=1$ for $i=1, \cdots, n$.

REMARK. It is easy to see that if $G$ is a noncompact locally compact group with property (E) and if $H$ is any locally compact group then the direct product $G \times H$ also has property (E). Many similar observations can be made.

There exist locally compact groups without property (E). We include three such examples here.

EXAMPLE 1. Let $G=S L(2, \mathrm{R})$, the group of two-by-two matrices over $\mathbf{R}$ with determinant 1 . (Note that $G$ is a nonamenable semisimple Lie group.) Since $G$ is a closed subset of $\mathbf{R}^{4}$, a set $X \subset G$ is relatively compact if and only if it is bounded with respect to a norm on $\mathrm{R}^{4}$. Let $X$ be a subset of $G$ with $\mathrm{cl} X$ being noncompact. Let $U$ be a neighborhood of the identity matrix $\delta$ in $G$. We shall prove that the set $D=\left\{\sigma^{-1} \tau \sigma: \sigma \in X, \tau \in U\right\}$ is unbounded. (It clearly implies that $X$ is not an $E$-set and hence $G$ fails to have property (E).) 
If $\tau \in G$ the components of $\tau$ will be denoted by $\tau_{i j}, i, j=1,2$. There exists $\epsilon>0$ such that the set $A=\left\{\alpha \in G: \alpha_{11}=\alpha_{22}=1,\left|\alpha_{12}\right|<\epsilon, \alpha_{21}=0\right\}$ and the set $B=\left\{\beta \in G: \beta_{11}=\beta_{22}=1, \beta_{12}=0,\left|\beta_{21}\right|<\epsilon\right\}$ are contained in $U$. For $\alpha \in A, \beta \in B$ write $\sigma^{-1} \alpha \sigma=\left(a_{i j}\right)$ and $\sigma^{-1} \beta \sigma=\left(b_{i j}\right)$. Then

$$
\begin{array}{ll}
a_{12}=\sigma_{22}^{2} \alpha_{12}, & a_{21}=-\sigma_{21}^{2} \alpha_{12}, \\
b_{12}=-\sigma_{12}^{2} \beta_{21}, & b_{21}=\sigma_{11}^{2} \beta_{21} .
\end{array}
$$

Let $X_{i j}=\left\{\sigma_{i j}: \sigma_{i j}\right.$ is the $(i, j)$ th component of some $\left.\sigma \in X\right\}, i, j=1,2$. Since $X$ is unbounded, at least one of the sets $X_{i j}$ is unbounded. By (1) the set $D$ is unbounded as we claimed.

ExAmple 2. Let $G=\mathbf{C} \times \mathbf{T}$ with product topology and with multiplication given by

$$
(z, w)\left(z^{\prime}, w^{\prime}\right)=\left(z+w z^{\prime}, w w^{\prime}\right) . .
$$

(Note that $G$ is a solvable Lie group and $G / C=T$ is compact and abelian.) Note that a set $X \subset G$ is not relatively compact if and only if the collection of first coefficients of elements in $X$ is unbounded in C. Suppose that $U$ is a neighborhood of $(0,1)$ in $G$ and $\operatorname{cl} X$ is noncompact. Then the set

$$
\begin{aligned}
\left\{(z, w)^{-1}\right. & (a, b)(z, w):(z, w) \in X,(a, b) \in U\} \\
& =\{(\bar{w}(-z+a+b z), b):(z, w) \in X,(a, b) \in U\}
\end{aligned}
$$

is clearly not relatively compact. Therefore $G$ fails to have property (E).

ExAmple 3. Let $G=\mathbf{R} \times \mathbf{R} \times \mathbf{T}$ with the product topology and with multiplication given by

$$
(x, y, \exp (i \theta))\left(x^{\prime}, y^{\prime}, \exp \left(i \theta^{\prime}\right)\right)=\left(x+x^{\prime}, y+y^{\prime}, \exp \left(i\left(\theta+\theta^{\prime}+x y^{\prime}\right)\right)\right) .
$$

(Note that $G$ is a nilpotent Lie group with center $\{0\} \times\{0\} \times$ T.) Let $U=$ $\mathbf{R} \times \mathbf{R} \times\{\exp (i \theta):|\exp (i \theta)-1|<1\}$. Then $U$ is a neighborhood of $(0,0,1)$. It is not hard to see that if $X \subset G$ is not relatively compact and if $W$ is a neighborhood of $(0,0,1)$ then

$$
D=\left\{t^{-1} w t: t \in X, w \in W\right\} \not \subset U .
$$

Indeed, the set $\{\exp (i \theta)$ : there exists $x, y \in \mathbf{R}$ with $(x, y, \exp (i \theta)) \in D\}=\mathbf{T}$. Therefore $G$ fails to have property (E).

One of the reasons that we are interested in groups with property $(E)$ is that we have the following. 
that $U$ is a compact neighborhood of $e$ such that $x U \cap x^{\prime} U=\varnothing$ if $x, x^{\prime} \in X$, $x \neq x^{\prime}$. Let $f \in C(G)$ with its support contained in $U$ and let $\left\{c_{x}\right\}_{x \in X}$ be a bounded set of complex numbers indexed by $X$. Then the function $g=$ $\Sigma\left\{c_{x^{-1}} l_{x^{-1}} f: x \in X\right\}$ belongs to $U C(G)$.

Proof. Note first that $g$ is well defined since for each $y \in G$ there is at most one $x \in X$ with $\left(l_{x^{-1}} f\right)(y) \neq 0$. Let $\epsilon>0$ be given. Since $f$ is uniformly continuous, there exists a neighborhood $V$ of $e$ such that $V \subset U$ and $|f(y)-f(z)|<\epsilon$ if either $y z^{-1} \in V$ or $z^{-1} y \in V$. For each $y \in G$ and $a \in V$ at most two terms in $r_{a} g-g=\Sigma\left\{c_{x}\left(f\left(x^{-1} y a\right)-f\left(x^{-1} y\right)\right): x \in X\right\}$ can be different from zero. Note that $\left(x^{-1} y\right)^{-1}\left(x^{-1} y a\right)=a \in V$. Therefore $\left\|r_{a} g-g\right\|_{\infty} \leqslant 2 \epsilon \cdot \sup \left\{\left|c_{x}\right|: x \in X\right\}$ if $a \in V$. So $g$ is left uniformly continuous.

Let $\epsilon$ and $V$ be as in the previous paragraph. Since $X$ is an $E$-set, there is a neighborhood $W$ of $e$ such that $W \subset x V x^{-1}, x \in X$. If $a \in W, x \in X$, $y \in G$ then $\left(x^{-1} a y\right)\left(x^{-1} y\right)^{-1}=x^{-1} a x \in V$. Therefore $\left\|l_{a} g-g\right\|_{\infty} \leqslant 2 \epsilon \cdot$ $\sup \left\{\left|c_{x}\right|: x \in X\right\}$. So $g$ is right uniformly continuous.

Now we are ready to state and prove one of our main results.

THEOREM 4.6. Let $G$ be a noncompact locally compact group with prop-

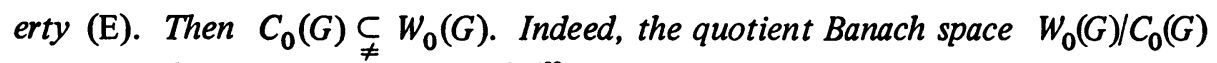
contains a linear isometric copy of $l^{\infty}$ and hence is nonseparable.

Proof. Let $X$ be a fixed relatively noncompact symmetric $E$-set in $G$. Let $V$ be a fixed compact symmetric neighborhood of the identity $e$. Choose a compact symmetric neighborhood $W$ of $e$ such that $x^{-1} W x \subset V$ if $x \in X$ and $W \subset V$. Choose $x_{1} \in X$. For $n \geqslant 2$, choose $x_{n}$ inductively such that $x_{n} \in X$ and

$$
x_{n} \notin\left\{\prod_{i=1}^{7} z_{i}: z_{i} \in V \cup\left\{x_{1}, \cdots, x_{n-1} ; x_{1}^{-1}, \cdots, x_{n-1}^{-1}\right\}\right\} .
$$

Set $T=\bigcup_{k=1}^{\infty} x_{k} W$. We claim that

(1) $x_{n} V \cap x_{m} V=\varnothing$ if $n \neq m$,

(2) $T \cap T y$ is compact if $y \notin V^{2}$,

(3) $T \cap y T$ is compact if $y \notin V^{2}$.

(1) holds since $x_{n} \notin\left\{x_{1}, \cdots, x_{n-1}\right\} V^{2}$. To see (2), let $y \in G, y \notin V^{2}$. Suppose that $T \cap T y$ is nonempty. (Otherwise there is nothing to be proved.) Fix $x_{n} a=x_{m} b y \in T \cap T y$ where $a, b \in W$. Since $y \notin V^{2}, n$ has to be different from $m$. Let $k=\max \{m, n\}$. Let $x_{n^{\prime}} a^{\prime}=x_{m^{\prime}} b^{\prime} y$ be any given point in $T \cap T y$ where $a^{\prime}, b^{\prime} \in W$. Then $n^{\prime} \neq m^{\prime}$. We claim that $k^{\prime}=$ $\max \left\{n^{\prime}, m^{\prime}\right\} \leqslant k$. If not, say $k^{\prime}=n^{\prime}>k$ then $x_{n^{\prime}}=x_{n^{\prime}} b^{\prime} b^{-1} x_{m}^{-1} x_{n} a a^{\prime-1}$, contradicting (*). Therefore, 


$$
T \cap T y \subset x_{1} W \cup \cdots \cup x_{k} W
$$

and hence is compact.

To see (3), let $y \notin V^{2}$ and suppose that $x_{n} w=y x_{m} w_{1} \in T \cap y T$ where $w, w_{1} \in W$. We claim that $n \neq m$. If $n=m$, then $y=x_{n} w w_{1}^{-1} x_{n}^{-1}=$ $x_{n} w x_{n}^{-1} x_{n} w_{1}^{-1} x_{n}^{-1} \in V^{2}$, a contradiction. The remaining part of the proof is the same as the proof of (2). (The construction of the set $T$ is a generalization of Lemma 1 in [2].)

Pick $h \in C(G)$ such that $h(e)=1,0 \leqslant h \leqslant 1$ and $h \equiv 0$ off $W$. Set

$$
f(x)=\sum_{n=1}^{\infty} h\left(x_{n}^{-1} x\right), \quad x \in G .
$$

We claim that $f \in W_{0}(G) \backslash C_{0}(G)$. By Lemma $4.5, f \in U C(G)$ and clearly $0 \leqslant$ $f \leqslant 1$. Note that $f\left(x_{n}\right)=1$ for $n=1,2, \cdots$ and that $\left\{x_{1}, x_{2}, \cdots\right\}$ is not relatively compact (by (1)). Therefore $f \notin C_{0}(G)$. It remains to show that $f$ is w.a.p. and $m(f)=0$.

To show that $f$ is w.a.p. we shall follow the steps in [14, Lemma 3] quite closely. We shall prove that if $\left(a_{n}\right)$ is a sequence in $G$ then there is a subsequence $\left(a_{n_{k}}\right)$ such that $\left(l_{a_{n_{k}}} f\right)$ converges pointwisely on $\beta G$ to a continuous function $g$ on $\beta G$.

Case 1. Some compact subset of $G$ contains infinitely many $a_{i}$. Since $f$ is right uniformly continuous, some subsequence of $\left(l_{a_{n}} f\right)$ converges uniformly and hence weakly.

Case 2. No compact subset of $G$ contains infinitely many $a_{i}$. Then there is a subsequence of $\left(a_{n}\right)$ which is again denoted by $\left(a_{n}\right)$ such that

$$
a_{i} a_{j}^{-1} \notin V^{2} \text { if } i \neq j \text {. }
$$

Suppose that $i \neq j$. If $\left(l_{a_{i}} f\right)(y) \cdot\left(l_{a_{j}} f\right)(y) \neq 0$ then $y \in a_{i}^{-1} T \cap a_{j}^{-1} T$. Therefore, by (3) and (4), we know that the support of $l_{a_{i}} f \cdot l_{a_{j}} f$ is compact and hence $l_{a_{i}} f \cdot l_{a_{j}} f \equiv 0$ on $\beta G \backslash G$. Thus $\lim l_{a_{i}} f(y)=0$ if $y \in \beta G \backslash G$. So it remains to show that some subsequence of $\left(l_{a_{i}} f\right)$ converges pointwisely on $G$ to a continuous function on $G$ with compact support. If $\lim _{i}\left(l_{a_{i}} f\right)(y)=0$ for each $y \in G$ then the proof is completed. So assume that for some $z \in G$, lim $\sup _{i} f\left(a_{i} z\right)>0$. By taking a subsequence if necessary, we may assume that $f\left(a_{i} z\right) \neq 0$ for each $i$. Let $y \in G$ such that $f\left(a_{i} y\right) \neq 0$ for infinitely many $i$. Then $y \in a_{i}^{-1} T$ for infinitely many $i$. Say, $z=a_{i}^{-1} p_{i}, y=a_{i}^{-1} q_{i}$ for $i \in I$, an infinite subset of the set of positive integers, where $p_{i}, q_{i} \in T$. Then $p_{i}=$ $q_{i} y^{-1} z \in T \cap T y^{-1} z, i \in I$. Since $\left\{a_{i}: i \in I\right\}$ is not contained in a compact subset of $G,\left\{p_{i}: i \in I\right\}$ is not either. By (2), $y^{-1} z \in V^{2}$, i.e., $y \in z V^{2}$. So 


$$
\lim _{n}\left(l_{a_{n}} f\right)(y)=0 \text { if } y \notin z V^{2} .
$$

Since $f$ is uniformly continuous, $\left(l_{a_{n}} f\right)$ is a bounded equicontinuous family of functions on the compact set $z V^{3}$. By the Arzela-Ascoli Theorem, there is a subsequence of $\left(l_{a_{n}} f\right)$, say, $\left(l_{a_{n_{k}}} f\right)$ which converges uniformly on $z V^{3}$ to a continuous function $g$ on $z V^{3}$. Note that $g \equiv 0$ on $z V^{3} \backslash z V^{2}$, by (5). Extend $g$ to $G$ by setting $g \equiv 0$ off $z V^{3}$. Then $\lim _{k} l_{a_{n_{k}}} f=g$ pointwisely on $G$ and $g$ is continuous and is with compact support. Therefore we have finished the proof of the fact that $f$ is w.a.p.

To see $m(f)=0$, for each $n$ pick $b_{1}, \cdots, b_{n}$ in $G$ such that $b_{i} b_{j}^{-1}$ $\notin V^{2}$ if $i \neq j$. Note that if $\left(l_{b_{1}} f+\cdots+l_{b_{n}} f\right)(y)>1$ then $y \in$ $\bigcup\left\{b_{i}^{-1} T \cap b_{j}^{-1} T: i \neq j, 1 \leqslant i, j \leqslant n\right\} \equiv A$. Note that $A$ is compact, by (2). Choose $\xi \in C(G)$ with compact support such that $\xi \equiv 1$ on $A, 0 \leqslant \xi \leqslant 1$. Then

$$
1+n \xi \geqslant l_{b_{1}} f+\cdots+l_{b_{n}} f .
$$

Therefore, $m(1)+n m(\xi) \geqslant n m(f)$. Since $m(\xi)=0, m(f) \leqslant 1 / n$. But $n$ can be arbitrarily large, $m(f)=0$, i.e., $f \in W_{0}(G)$.

Now let us prove that $W_{0}(G) \backslash C_{0}(G)$ contains a linear isometric copy of $l^{\infty}$. Write $\left\{x_{n}: n=1,2, \cdots\right\}$ as a disjoint union of a countably infinite family of sets $Y_{k}$ with each $Y_{k}$ being infinite (and hence relatively noncompact). Let $f_{k}=\Sigma\left\{l_{x-1} h: x \in Y_{k}\right\}$. Then as above $f_{k} \in W_{0}(G) \backslash C_{0}(G)$. Indeed, if $\left(c_{k}\right)^{\cdot}$ is a nonzero element in $l^{\infty}$ then $\Sigma_{k} c_{k} f_{k} \in W_{0}(G) \backslash C_{0}(G)$ by using the same proof as the proof of the fact that $f \in W_{0}(G) \backslash C_{0}(G)$. Since the support of each $f_{k}$ is noncompact, one sees that

$$
\begin{aligned}
\left\|\sum_{k} c_{k} f_{k}+C_{0}(G)\right\| & \equiv \inf \left\{\left\|\sum_{k} c_{k} f_{k}+g\right\|_{\infty}: g \in C_{0}(G)\right\} \\
& =\left\|\sum_{k} c_{k} f_{k}\right\|_{\infty}=\left\|\left(c_{k}\right)\right\|_{\infty} .
\end{aligned}
$$

Therefore, the mapping from $l^{\infty}$ into $W_{0}(G) / C_{0}(G)$ which sends $\left(c_{k}\right) \in l^{\infty}$ into $\Sigma c_{k} f_{k}+C_{0}(G)$ is a linear isometry and the proof of the theorem is completed.

REMARK. Let $G$ be a locally compact group. Suppose that there exists a sequence of functions $f_{k} \in W_{0}(G), k=1,2, \cdots$, such that (i) the supports of the functions $f_{k}$ are mutually disjoint, (ii) $0 \leqslant f_{k} \leqslant 1$, (iii) the set $\{x \in G$ : $\left.f_{k}(x)=1\right\}$ is noncompact, and (iv) for each $\left(c_{k}\right) \in l^{\infty}, \Sigma_{k} c_{k} f_{k} \in W_{0}(G)$. Then 
the mapping from $l^{\infty}$ into $W_{0}(G) / C_{0}(G)$ defined by sending $\left(c_{k}\right)$ to $\Sigma_{k} c_{k} f_{k}$ $+C_{0}(G)$ is a linear isometry. In the last paragraph of the above proof we have established the existence of such a sequence $\left(f_{k}\right)$ for a noncompact group with property (E). Note that if $\varphi$ is a continuous homomorphism from a locally compact group $K$ onto $G$ where $G$ has a sequence of functions $f_{k} \in W_{0}(G)$ which satisfies (i) to (iv) then the sequence of functions $f_{k} \circ \varphi \in W_{0}(K)$ also satisfies conditions (i) to (iv).

COROLLARY 4.7. If $G$ is a noncompact locally compact nilpotent group then the quotient Banach space $W_{0}(G) / C_{0}(G)$ contains a linear isometric copy of $l^{\infty}$.

Proof. It is enough to prove the following assertion: For each noncompact locally compact nilpotent group $G$ there is a sequence of functions $f_{k} \in$ $W_{0}(G)$ which satisfies conditions (i) to (iv) in the above remark. Since $G$ is nilpotent, the upper central sequence is finite: $G=G_{0} \supset G_{1} \supset \cdots \supset G_{n-1} \supset$ $G_{n}=(e)$ where each $G_{i}$ is closed and $G_{i-1} / G_{i}$ is the center of $G / G_{i}, i=1$, $2, \cdots, n$. We shall call $n$ the rank of $G$.

If $n=1$, then $G$ is abelian and our assertion is a special case of Theorem 4.6. Assume that our assertion holds if the rank of the group is $n-1(n \geqslant 2)$. Let $G$ be noncompact and be of rank $n$. If $G_{n-1}$, the center of $G$, is noncompact, then $G$ has property (E) (Proposition 4.4(2)) and hence our assertion is again a special case of Theorem 4.6. If $G_{n-1}$ is compact, then $G / G_{n-1}$ is noncompact and is of rank $n-1$. Therefore, by inductive assumption, our assertion holds for $G / G_{n-1}$ and hence it also holds for $G$ by the remark preceding this corollary.

REMARKS. (1) The conclusion in Corollary 4.2 can be strengthened as in the above corollary.

(2) By Lemma 4.1 it is quite clear that property (E) is not a necessary condition for $C_{0}(G)$ to be contained in $W_{0}(G)$ properly. For example, if $G=$ $S L(2, \mathbf{R}) \times S L(2, \mathbf{R})$ (direct product) then by Lemma $4.1(2), C_{0}(G) \underset{\ddagger}{\subsetneq} W_{0}(G)$ while it is not hard to see that $G$ fails to have property (E) (cf. Example 1). Other such examples are the group in Example 3 and the group $A_{n}$ of affine mappings of $\mathbf{R}^{n}$ onto $\mathbf{R}^{n}$. Indeed, $W_{0}\left(A_{n}\right) / C_{0}\left(A_{n}\right)$ contains a linear isometric copy of $l^{\infty}$.

THEOREM 4.8. Let $G=\mathbf{C} \times \mathbf{T}$ be the group defined in Example 2. Then $W_{0}(G)=C_{0}(G)$.

Proof. Let $f \in U C(G)$ be fixed such that $f \geqslant 0$ and $f \in F R_{0}(G) \backslash C_{0}(G)$. If we can conclude that $f$ is not w.a.p. then the proof of the theorem is completed. Toward this end, note first that since $f \notin C_{0}(G)$ there exist $\alpha>0, z_{n}=$ 
$\rho_{n} \exp \left(i \theta_{n}\right)$ with $\rho_{n} \rightarrow+\infty$ and $\exp \left(i \varphi_{n}\right) \in \mathrm{T}$ such that $f\left(z_{n}, \exp \left(i \varphi_{n}\right)\right) \geqslant$ $3 \alpha$ for each $n$. By choosing a subsequence if necessary, we may assume that $\exp \left(i \theta_{n}\right)$ converges to some $\exp \left(i \theta_{0}\right) \in \mathbf{T}$ and $\exp \left(i \varphi_{n}\right)$ converges to some $\exp \left(i \varphi_{0}\right) \in \mathbf{T}$. Note that

$$
\begin{gathered}
\left.\left.\left.{ }^{\left[r_{\left(0, \exp \left(i \left(\left(\varphi_{n}-\varphi_{0}\right)+\right.\right.\right.}\right.}+\left(\theta_{0}-\theta_{n}\right)\right)\right)\right) \\
=f\left(0, \exp \left(i\left(\theta_{n}-\theta_{0}\right)\right)\right) \\
=f]\left(z_{n}, \exp \left(i \varphi_{n}\right)\right) .
\end{gathered}
$$

Since $f$ is uniformly continuous, the above relation implies that

$$
\left|f\left(\rho_{n} \exp \left(i \theta_{0}\right), \exp \left(i \varphi_{0}\right)\right)-f\left(z_{n}, \exp \left(i \varphi_{n}\right)\right)\right|
$$

is small when $n$ is large. Therefore, we may assume that $f\left(\rho_{n} \exp \left(i \theta_{0}\right), \exp \left(i \varphi_{0}\right)\right)$ $\geqslant 2 \alpha$ for each positive integer $n$. By translating $f$ by $r_{\left(0, \exp i\left(\varphi_{0}-\theta_{0}\right)\right)}{ }^{l}\left(0, \exp \left(i \theta_{0}\right)\right)$ we may assume that $\theta_{0}=0$ and $\varphi_{0}=0$. So we have the following:

$$
f\left(\rho_{n}, 1\right) \geqslant 2 \alpha \text { for } n=1,2, \cdots \text {, and } \rho_{n} \rightarrow+\infty \text {. }
$$

For the sake of clarity, we single out part of the proof as

LEMMA 4.9. For two positive real numbers $c$ and $\beta$ set

$$
A_{c, \beta}=\{y \in \mathbf{R}: y \geqslant 0, f(c+i y, 1) \geqslant \beta\} .
$$

Then $D\left(A_{c, \beta}\right) \equiv \lim \sup _{n}\left\{\sup \left\{n^{-1} \lambda_{\mathbf{R}}\left([y, n+y] \cap A_{c, \beta}\right): y \in \mathbf{R}, y \geqslant 0\right\}\right\}=0$.

Proof. Suppose that $D\left(A_{c, \beta}\right)>0$ for some $c>0$ and some $\beta>0$. We want to get a contradiction.

If $s$ and $t$ are fixed positive numbers, then, since $d=D\left(A_{c, \beta}\right)>0$, it is not hard to see that there exists a positive number $y_{0}$ such that $y_{0}>t$ and

$$
(1 / s) \lambda_{\mathbf{R}}\left(\left[y_{0}, y_{0}+s\right] \cap A_{c, \beta}\right) \geqslant d / 2 .\left(^{4}\right)
$$

Since $f$ is uniformly continuous there exists $\delta>0$ such that if $|w|<\delta$, $|\exp (i \theta)-1|<\delta$ and $|\exp (i \varphi)-1|<\delta$ then

$$
|f(z, 1)-f(z \exp (i \theta)+w, \exp (i \varphi))|<\beta / 2 .\left({ }^{5}\right)
$$

(4) Choose $n>5(t+s) / d$ such that $n^{-1} \lambda_{\mathrm{R}}\left([y, y+n] \cap A_{c, \beta}\right) \geqslant 3 d / 4$ for some $y>0$. Choose a positive integer $k$ such that $y+t+k s<y+n$ and $y+t+(k+1) s\rangle$ $y+n$. Then there exists a $j, 0<j<k-1$, such that

$$
s^{-1} \lambda_{\mathbf{R}}\left([y+t+j s, y+t+(j+1) s] \cap A_{c, \beta}\right) \geq d / 2 \text {. }
$$

If not then $n^{-1} \lambda_{\mathrm{R}}\left([y, y+n] \cap A_{c, \beta}\right) \leqslant n^{-1}(t+k s d / 2+s)<d / 5+d / 2<3 d / 4$, a contradiction.

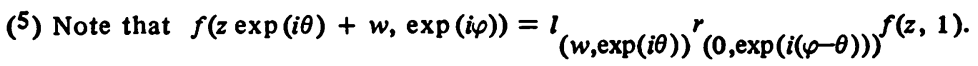


Let $s$ be a fixed positive number. For a given large positive number $t$ denote the unique complex number with absolute value $t$ and with real part $c+s$ by $w_{0}$. Choose $t$ so large such that (i) if $\psi$ is the angle formed by the imaginary axis and the ray connecting 0 and $w_{0}$ then $|\exp (i \psi)-1|<\delta$ and (ii) $t$-imaginary part of $w_{0}<\delta$. Choose $y_{0}>t$ such that (2) holds. Note that by the ways $t$ and $y_{0}$ are chosen and by (3) we have

$$
f(x+i y, \exp (i \varphi)) \geqslant \beta / 2 \text { if } y \in A_{c, \beta} \cap\left[y_{0}, y_{0}+s\right],
$$

$c \leqslant x \leqslant c+s$, and $|\exp (i \varphi)-1|<\delta$.

Note that $G$ is the topological semidirect product of $\mathbf{C}$ and $\mathbf{T}$ where the action of $\mathbf{T}$ on $\mathbf{C}$ is given by $\exp (i \varphi): z \rightarrow \exp (i \varphi) \cdot z$. Therefore $G$ is unimodular, and the Haar measure of $G$ is just the product of the Lebesgue measure for $\mathbf{C}$ and the normalized Lebesgue measure for $T$, cf. [11, p. 210]. For each positive integer $n$, let

$$
U_{n}=\{x+i y=|x| \leqslant n / 2, \quad|y| \leqslant n / 2\} \times \mathbf{T} .
$$

It is easy to see that $\left(U_{n}\right)$ is an $F$-sequence with respect to multiplication from the right and that $\lambda\left(U_{n}\right)=n^{2}$. Since $f \in F R_{0}(G)$, by the right-hand version of Lemma 2.3, we have

$$
\lim _{n}\left(1 / n^{2}\right) \int_{U_{n}} f(a u) d u=0 \text { uniformly in } a(a \in G) .
$$

Let $s=n$, a positive integer. Choose $y_{0}$ as above and let $a=((c+n / 2)$ $\left.+i\left(y_{0}+n / 2\right), 1\right)$. By (2) and (4) the Haar measure of the set $\left\{v \in a U_{n}: f(v) \geqslant \beta / 2\right\}$ is $\geqslant n \cdot(d / 2) \cdot n \cdot(\delta / \pi)$. Therefore

$$
\begin{aligned}
\left(1 / n^{2}\right) \int_{U_{n}} f(a u) d u & =\left(1 / n^{2}\right) \int_{a U_{n}} f(u) d u \\
& \geqslant\left(1 / n^{2}\right) \cdot n^{2} \cdot(d / 2) \cdot(\delta / \pi) \cdot(\beta / 2)=d \delta \beta / 4 \pi>0 .
\end{aligned}
$$

The above relation certainly contradicts (5) and hence the lemma is proved.

Now let us go back to continue the proof of the theorem.

We claim that it is possible to pick a sequence of positive integers $n_{1}<n_{2}$ $<\cdots$, and a sequence of nonnegative real numbers $y_{1}, y_{2}, \cdots$ such that, for each $j$,

$$
\begin{aligned}
& f\left(\rho_{n_{j}}+i y_{p}, 1\right) \geqslant \alpha, \quad p=1,2, \cdots, j, \\
& f\left(\rho_{n_{j}}+i y_{p}, 1\right) \leqslant \alpha / 2, \quad p=j+1, j+2, \cdots .
\end{aligned}
$$

Choose $n_{1}=1$ and $y_{1}=0$. Then by (1) $f\left(\rho_{n_{1}}+i y_{1}, 1\right) \geqslant 2 \alpha \geqslant \alpha$. Suppose we have picked $n_{1}<\cdots<n_{k}$ and $y_{1}, \cdots, y_{k}$ such that (6) holds for $j \leqslant k$ and (7) holds for $j \leqslant k$ and $p \leqslant k$. Let 


$$
A_{k}=A_{\rho_{n_{1}}, \alpha / 2} \cup \cdots \cup A_{\rho_{n_{k}}, \alpha / 2} .
$$

By Lemma 4.9, $D\left(A_{k}\right)=0$. Therefore there exists $y_{k+1} \geqslant 0, y_{k+1} \notin A_{k}$, i.e., $f\left(\rho_{n_{j}}+i y_{k+1}, 1\right) \leqslant \alpha / 2$ for $j=1,2, \cdots, k$. Again note that $f$ is uniformly continuous and $\rho_{n} \rightarrow+\infty$. If $s$ is a fixed positive number then $\mid f\left(\rho_{n}, 1\right)$ $-f\left(\rho_{n}+i y, 1\right) \mid$ becomes uniformly (in $y, 0 \leqslant y \leqslant s$ ) close to zero when $n$ becomes large. Therefore, by (1) there exists $n_{k+1}>n_{k}$ such that $f\left(\rho_{n_{k+1}}+i y_{p}, 1\right)$ $\geqslant \alpha$ if $p=1,2, \cdots, k+1$. Thus, by induction, we know that our claim is true.

Let $f_{p}=l_{\left(i y_{p}, 1\right)} f, p=1,2, \cdots$. If $f$ is w.a.p. then there is a subsequence $f_{p_{j}}$ such that $\lim _{j} f_{p_{j}}=g$ pointwisely on $\beta G$ where $g$ is a continuous function on $\beta G$. Let $a$ be a cluster point of $\left\{\left(\rho_{n_{k}}, 1\right): k=1,2, \cdots\right\}$ in $\beta G$. Then by (6) $f_{p_{j}}(a) \geqslant \alpha$ for each $j$ and hence $g(a) \geqslant \alpha$. On the other hand, by (7), $g\left(\rho_{n_{k}}, 1\right)=\lim _{j} f\left(\rho_{n_{k}}+i y_{p_{j}}, 1\right) \leqslant \alpha / 2$ and hence $g(a) \leqslant \alpha / 2$. We have arrived at a contradiction and hence $f$ is not w.a.p.

REMARKS. (1) By the above theorem we know that in Corollary 4.7 the condition nilpotency cannot be replaced by solvability.

(2) In [6, Theorem 7.1] DeLeeuw and Glicksberg proved that if $G$ is a locally compact abelian group and $H$ a closed subgroup then $W(H)=W(G) \mid H$. Their theorem does not hold for the group $G=\mathbf{C} \times \mathbf{T}$ of Example 2 in the following dramatic way: $W(\mathbf{C}) \not \subset U C(G) \mid \mathbf{C}$. (Note that $\mathbf{C}$ is a normal subgroup of G.) For example, let $h \in C(\mathrm{C})$ with $h(0)=1$ and $h \equiv 0$ outside of the unit disc. Set $f(z)=\sum_{n=1}^{\infty} h\left(z-2^{n}\right), z \in \mathbf{C}$. Then $f \in W(\mathrm{C})$ and clearly $f$ is not the restriction of any uniformly continuous function on $G$.

(3) For a locally compact group $G$, denote the Fourier-Stieltjes algebra of $G$ by $B(G)$, cf. D. Eymard's L'algèbre de Fourier d'un groupe localement compact, Bull. Soc. Math. France 92 (1964), 181-236, for the definition. It is known that $B(G) \subset W(G)$. If $G$ is noncompact and abelian D. E. Ramirez proved that the uniform closure of $B(G)$ is a proper subset of $W(G)$, cf. [1] for a proof. However, if $G$ is the group in Theorem 4.8 then the uniform closure of $B(G)$, $B(G)^{-}$, equals $W(G)$ since $A(G) \subset B(G)^{-}$by Peter-Weyl Theorem and $C_{0}(G)$ $\subset B(G)^{-}$, cf. Eymard's paper quoted above.

5. $W_{0}(G)$ and $F_{0}(G)$. If $G$ is a noncompact locally compact amenable group then $W(G) \subsetneq F(G)$, cf. §3. A harder problem is to decide whether $W_{0}(G)$ $\subsetneq F_{0}(G)$. In [1, Theorem 3.19] Burckel proved that $W_{0}(\mathrm{R}) \underset{\ddagger}{\subsetneq} F_{0}(\mathrm{R})$. We have the following stronger result.

THEOREM 5.1. Let $G$ be a locally compact amenable group with a relatively noncompact $E$-set $X$ such that $\left\{x a x^{-1}: x \in X \cup X^{-1}\right\}$ is relatively compact 
for each $a \in G$. Then $F_{0}(G) / W_{0}(G)$ contains a linear isometric copy of $l^{\infty}$.

Proof. By replacing $X$ by $X \cup X^{-1}$ we shall assume that $X$ is symmetric. We shall also assume that $e \in X$. Note that if $K$ is a compact subset of $G$ then $\bigcup\left\{x K x^{-1}: x \in X\right\}$ is relatively compact. Indeed, pick any compact neighborhood $W$ of $e$ and choose a compact neighborhood $U$ of $e$ such that $x U x^{-1} \subset W$ for $x \in X$. Cover $K$ by, say, $a U, a U, \cdots, a_{k} U, a_{1}, \cdots, a_{k} \in G$. Then $\bigcup\left\{x K x^{-1}: x \in G\right\}$ is contained in

$$
\bigcup\left\{\left(x a_{1} x^{-1}\right)\left(x U x^{-1}\right) \cup \cdots \cup\left(x a_{k} x^{-1}\right)\left(x U x^{-1}\right): x \in X\right\}
$$

and hence is relatively compact.

Assume first that $G$ is $\sigma$-compact and unimodular. Then $G$ has an $F$-sequence $\left(U_{n}\right)$ such that each $U_{n}$ is, in addition, symmetric, cf. [3, Theorem 4.4]. Choose a compact symmetric neighborhood $V$ of $e$ such that $\lambda(V)<1$. For convenience, let $U_{0}=\varnothing$ and $n_{0}=0$.

Pick $x_{1}=y_{1}=e \in X$. Pick $n_{1}$ such that $x V^{2} x^{-1} \subset U_{n_{1}}$ for $x \in X$ and $\lambda\left(U_{n_{1}}\right) \geqslant 2$. Suppose that we have picked $x_{1}, \cdots, x_{k} ; y_{1}, \cdots, y_{k}$ from $X$ and positive integers $n_{1}<\cdots<n_{k}$ such that the following are satisfied:

(i) $x_{i} y_{j} V \subset U_{n_{p}} \backslash U_{n_{p-1}}$ if $1 \leqslant i, j \leqslant k, p=\max \{i, j\}$.

(ii) $x_{i} y_{j} V \cap x_{i^{\prime}} y_{j^{\prime}} V=\varnothing$ if $1 \leqslant i, i^{\prime}, j, j^{\prime} \leqslant k$ and $(i, j) \neq\left(i^{\prime}, j^{\prime}\right)$.

(iii) $x_{i} x_{i^{\prime}}^{-1}, x_{i}^{-1} x_{i^{\prime}} \notin U_{n_{i-1}}$ if $1 \leqslant i^{\prime}<i \leqslant k ;\left(y_{j} V\right)\left(y_{j^{\prime}} V\right)^{-1} \subset$ $G \backslash U_{n_{j-1}},\left(y_{j} V\right)^{-1}\left(y_{j^{\prime}} V\right) \subset G \backslash U_{n_{j-1}}$ if $1 \leqslant j^{\prime}<j \leqslant k$.

(iv) $V U_{n_{i-1}}^{2} V \subset U_{n_{i}}$ if $i=2, \cdots, k$.

(v) $\lambda\left(U_{n_{i}}\right) \geqslant 2^{i}$ if $i=1,2, \cdots, k$.

(vi) $x U_{n_{i-1}} x^{-1} \subset U_{n_{i}}, i=2, \cdots, k, x \in X$.

Pick $y_{k+1} \in X$ such that (a) $\left(y_{k+1} V\right)\left(y_{j} V\right)^{-1} \subset G \backslash U_{n_{k}},\left(y_{k+1} V\right)^{-1}\left(y_{j} V\right)$ $\subset G \backslash U_{n_{k}}$ if $j=1,2, \cdots, k$, (b) $x_{i} y_{k+1} V \cap x_{i^{\prime}} y_{j} V=\varnothing$ if $1 \leqslant i, i^{\prime}, j \leqslant k$, (c) $x_{i} y_{k+1} V \subset G \backslash U_{n_{k}}, i=1, \cdots, k$. Such a $y_{k+1}$ exists since each of the following sets is compact: $U_{n_{k}} y_{j} V^{2} \cup y_{j} V U_{n_{k}} V, j=1,2, \cdots, k ; x_{i}^{-1} x_{i^{\prime}} y_{j} V^{2}$, $1 \leqslant i, j, i^{\prime} \leqslant k ; x_{i}^{-1} U_{n_{k}} V, i=1, \cdots, k$. Note that the condition $x_{i} y_{k+1} V \cap$ $x_{i^{\prime}} y_{k+1} V=\varnothing$ is automatically satisfied if $1 \leqslant i, i^{\prime} \leqslant k, i \neq i^{\prime}$. Indeed, if $x_{i} y_{k+1} v=x_{i^{\prime}} y_{k+1} v^{\prime} \in x_{i} y_{k+1} V \cap x_{i^{\prime}} y_{k+1} V$ where $v, v^{\prime} \in V$, then $x_{i^{\prime}}^{-1} x_{i}=$ $y_{k+1} v^{\prime} v^{-1} y_{k+1}^{-1} \in U_{n_{1}}$, contradicting (iii).

Pick $x_{k+1} \in X$ such that

(a) $x_{k+1} x_{i}^{-1}, x_{k+1}^{-1} x_{i} \notin U_{n_{k}}, i=1, \cdots, k$,

(b) $x_{k+1} y_{j} V \cap x_{i} y_{j^{\prime}} V=\varnothing$ if $1 \leqslant i, j, j^{\prime} \leqslant k$,

(c) $x_{k+1} y_{j} V \subset G \backslash U_{n_{k}}, j=1,2, \cdots, k+1$. Note again that we have 


$$
\begin{aligned}
& x_{k+1} y_{j} V \cap x_{k+1} y_{j^{\prime}} V=\varnothing \text { if } 1 \leqslant j, j^{\prime} \leqslant k+1 \text { and } j \neq j^{\prime} . \\
& \text { Choose } n_{k+1}>n_{k} \text { such that } \\
& \text { (a) } x_{k+1}\left\{y_{1}, \cdots, y_{k+1}\right\} V \cup\left\{x_{1}, \cdots, x_{k}\right\} y_{k+1} V \subset U_{n_{k+1}} \text {, } \\
& \text { (b) } \lambda\left(U_{n_{k+1}}\right) \geqslant 2^{k+1} \\
& \text { (c) } V U_{n_{k}}^{2} V \subset U_{n_{k+1}}, \\
& \text { (d) } x U_{n_{k}} x^{-1} \subset U_{n_{k+1}}, x \in X .
\end{aligned}
$$

The reason that (d) is possible is given in the first paragraph of the proof. By induction, we get sequences $\left(x_{i}\right),\left(y_{j}\right),\left(n_{k}\right)$ such that (i)-(vi) are satisfied for each $k$. Since $\left(U_{n_{k}}\right)$ is again an $F$-sequence we shall write $U_{k}$ for $U_{n_{k}}$. Let $Z=\left\{x_{i} y_{j}: i, j=1,2, \cdots \cdot\right.$. It is easy to see that $Z$ is again an $E$-set. Choose $h \in C(G), 0 \leqslant h \leqslant 1, h(e)=1$ and $h \equiv 0$ off $V$. Let

$$
f=\sum\left\{\frac{i-j}{i+j} l_{\left.\left(x_{i} y_{j}\right)^{-1} h: i, j=1,2, \cdots\right\}}\right. \text {. }
$$

By Lemma 4.5 and (ii) above we know that $f \in U C(G)$. We claim that (I) $d(|f|)=0$ and (II) $\|f-g\|_{\infty} \geqslant\|f\|_{\infty}$ if $g \in W(G)$.

Proof OF (I). According to Lemma 2.3, to see that $d(|f|)=0$ it suffices to show that

$$
\lim _{n} \frac{1}{\lambda\left(U_{n}\right)} \int_{U_{n}}|f(t x)| d t=\lim _{n} \frac{1}{\lambda\left(U_{n}\right)} \int_{U_{n}}|f(x t)| d t=0
$$

uniformly on $G$. Let $A=\bigcup\left\{x_{i} y_{j} V: i, j=1,2, \cdots\right\}, A_{1}=\bigcup\left\{x_{i} y_{j} V: i \geqslant j\right\}$ and $A_{2}=\bigcup\left\{x_{i} y_{j} V: i \leqslant j\right\}$. Note that the support of $f$ is contained in $A$ and that $A=A_{1} \cup A_{2}$. Therefore,

$$
\begin{aligned}
& \frac{1}{\lambda\left(U_{n}\right)} \int_{U_{n}}|f(t x)| d t \leqslant \frac{\lambda\left(U_{n} \cap A x^{-1}\right)}{\lambda\left(U_{n}\right)}, \\
& \frac{1}{\lambda\left(U_{n}\right)} \int_{U_{n}}|f(x t)| d t \leqslant \frac{\lambda\left(U_{n} \cap x^{-1} A\right)}{\lambda\left(U_{n}\right)},
\end{aligned}
$$

and hence to prove (I) it suffices to show that

$$
\lim _{n} \frac{\lambda\left(U_{n} x \cap A\right)}{\lambda\left(U_{n}\right)}=\lim _{n} \frac{\lambda\left(x U_{n} \cap A\right)}{\lambda\left(U_{n}\right)}=0
$$

uniformly on $G$. Note that

$$
\begin{aligned}
x U_{n} \cap A= & \left(U_{n} \cap x U_{n} \cap A\right) \cup\left(\left(U_{n+1} \backslash U_{n}\right) \cap x U_{n} \cap A\right) \\
& \cup\left(\left(U_{n+2} \backslash U_{n+1}\right) \cap x U_{n} \cap A\right) \cup \cdots .
\end{aligned}
$$


Suppose that $\left(U_{k} \backslash U_{k-1}\right) \cap x U_{n} \neq \varnothing$ for some $k>n$. Then $x \in U_{k+1}$ and hence $x U_{n} \subset U_{k+2}$. Therefore $\left(U_{k^{\prime}} \backslash U_{k^{\prime}-1}\right) \cap x U_{n}=\varnothing$ if $k^{\prime} \geqslant k+3$. Thus:

(2) There exist at most three $k$ 's with $k>n$ and $\left(U_{k} \backslash U_{k-1}\right) \cap x U_{n} \neq \varnothing$.

Let $k>n$ be fixed and set $T_{1}=\left(U_{k} \backslash U_{k-1}\right) \cap x U_{n} \cap A_{1}$ and $T_{2}=$ $\left(U_{k} \backslash U_{k-1}\right) \cap x U_{n} \cap A_{2}$. Note that $T_{1} \cup T_{2}=\left(U_{k} \backslash U_{k-1}\right) \cap x U_{n} \cap A$. Suppose that $T_{1} \neq \varnothing$ and let $x t=x_{k} y_{j} v \in T_{1}$ where $j \leqslant k, v \in V, t \in U_{n}$. (Note that each element in $U_{k} \backslash U_{k-1} \cap A$ is of the form $x_{k} y_{j} v$, by (i).) If there is no element in $T_{1}$ which can be expressed in the form $x_{k} y_{j} v^{\prime}$ with $j^{\prime} \leqslant k, j^{\prime} \neq j$ and $v^{\prime} \in V$ then $T_{1} \subset x_{k} y_{j} V$ and hence $\lambda\left(T_{1}\right)<1$. If there exists $j^{\prime} \neq j, v^{\prime} \in V$ and $t^{\prime} \in U_{n}$ such that $x t^{\prime}=x_{k} y_{j^{\prime}} v^{\prime} \in T$. Then, by (iv), $y_{j^{\prime}}^{-1} y_{j}=v^{\prime} t^{\prime-1} t v^{-1} \in V U_{n}^{2} V \subset U_{n+1}$. By (iii) $j, j^{\prime} \leqslant n+2$. Therefore, in this case, $T_{1}$ is contained in the union of at most $n+2$ left translates of $V$. Therefore $\lambda\left(T_{1}\right) \leqslant n+2$ is always true.

Suppose that $T_{2} \neq \varnothing$ and let $x t=x_{i} y_{k} v \in T_{2}$ where $t \in U_{n}, 1 \leqslant i \leqslant k$ and $v \in V$. If there exists $i^{\prime} \neq i, t^{\prime} \in U_{n}, v^{\prime} \in V$ such that $x t^{\prime}=x_{i} y_{k} v^{\prime} \in T_{2}$ then $x_{i}^{-1} x_{i^{\prime}}=y_{k} v t^{-1} t^{\prime} v^{\prime-1} y_{k}^{-1} \in y_{k} V U_{n}^{2} V y_{k}^{-1} \subset y_{k} U_{n+1} y_{k}^{-1} \subset U_{n+2}$ by (iv) and (vi). Therefore, $i, i^{\prime} \leqslant n+3$, by (iii). We have shown that $\lambda\left(T_{2}\right) \leqslant$ $n+3$ is always true.

By the above two paragraphs we see that

$$
\lambda\left(\left(U_{k} \backslash U_{k-1}\right) \cap x U_{n} \cap A\right) \leqslant 2 n+5 \text { if } k>n .
$$

Combining (1), (2) and (3), we conclude that

$$
\lambda\left(x U_{n} \cap A\right) \leqslant n^{2}+3 \cdot(2 n+5) .
$$

Therefore, for each $x \in G$,

$$
\lambda\left(x U_{n} \cap A\right) / \lambda\left(U_{n}\right) \leqslant\left(1 / 2^{n}\right)\left(n^{2}+3 \cdot(2 n+5)\right) \rightarrow 0 \text { as } n \rightarrow \infty .
$$

Similarly, for a fixed $k>n$, set $S_{1}=\left(U_{k} \backslash U_{k-1}\right) \cap U_{n} x \cap A_{1}$ and $S_{2}=$ $\left(U_{k} \backslash U_{k-1}\right) \cap U_{n} x \cap A_{2}$. If $t x=x_{k} y_{j} v, t^{\prime} x=x_{k} y_{j^{\prime}} v^{\prime} \in S_{1}$ where $t, t^{\prime} \in$ $U_{n}, j \neq j^{\prime}, v, v^{\prime} \in V$ then $\left(y_{j^{\prime}} v^{\prime}\right)\left(y_{j} v\right)^{-1}=x_{k}^{-1} t^{\prime} t^{-1} x_{k} \in U_{n+2}$ by (iv) and (vi). Therefore $j^{\prime}, j \leqslant n+3$ by (iii). If $t x=x_{i} y_{k} v=x_{i^{\prime}} y_{k} v^{\prime} \in S_{2}$ where $t, t^{\prime} \in U_{n}, i<i^{\prime}, v, v^{\prime} \in V$ then $x_{i^{\prime}}=t^{\prime} t^{-1} x_{i} y_{k} v v^{\prime-1} y_{k}^{-1} \in U_{n}^{2} U_{i} U_{1} \subset$ $U_{n+2} \cup U_{i+2}$. Therefore $\lambda\left(S_{1}\right) \leqslant n+3$ and $\lambda\left(S_{2}\right) \leqslant n+2$. As before, we may conclude that

$$
\lambda\left(U_{n} x \cap A\right) / \lambda\left(U_{n}\right) \leqslant\left(1 / 2^{n}\right)\left(n^{2}+3 \cdot(2 n+5)\right) \rightarrow 0 \text { as } n \rightarrow \infty .
$$

We have finished the proof of (I).

Proof OF (II). Note that, since $f\left(x_{i} y_{j}\right)=(i-j) /(i+j),\|f\|_{\infty}=1$. Suppose that there exists $g \in W(G)$ with $\|f-g\|<1-2 \delta$ for some $\delta>0$. 
Then for a fixed $j, g\left(x_{i} y_{j}\right)$ has to be greater than $\delta$ when $i$ is sufficiently large. On the other hand, for a fixed $i, g\left(x_{i} y_{j}\right)$ has to be less than $-\delta$ when $j$ is sufficiently large. Since $g$ is w.a.p. there is a subsequence $\left(x_{i_{n}}\right)$ of $\left(x_{i}\right)$ such that $l_{x_{i_{n}}} g \equiv g_{n}$ converges weakly to a continuous function $g_{0}$ on $G$. Let $y$ be a cluster point of $\left\{y_{i}\right\}$ in $\beta G$. Since $g_{n}\left(y_{j}\right) \geqslant \delta$ when $n$ is large, $g_{0}\left(y_{j}\right) \geqslant \delta$ and hence $g_{0}(y) \geqslant \delta$. On the other hand $g_{n}(y) \leqslant-\delta$ for each $n$ and hence $g_{0}(y) \leqslant-\delta$. It is a contradiction and the proof of (II) is completed.

(III) Write

$$
\begin{aligned}
& \left\{x_{n}\right\}=\bigcup_{n=1}^{\infty} X_{n}, X_{n_{1}} \cap X_{n_{2}}=\varnothing \quad \text { if } n_{1} \neq n_{2} \text {, each } X_{n} \text { infinite; } \\
& \left\{y_{j}\right\}=\bigcup_{n=1}^{\infty} Y_{n}, Y_{n_{1}} \cap Y_{n_{2}}=\varnothing \quad \text { if } n_{1} \neq n_{2} \text {, each } Y_{n} \text { infinite. }
\end{aligned}
$$

Number the elements in each $X_{n}$ and each $Y_{n}$, say, $X_{n}=\left\{x_{n_{1}}, x_{n_{2}}, \cdots\right\}$ and $Y_{n}=\left\{y_{n_{1}}, y_{n_{2}}, \cdots\right\}$. Let

$$
f_{n}=\sum\left\{\frac{i-j}{i+j} l\left(x_{n_{i}} y_{n_{j}}\right)^{-1} h: i, j=1,2, \cdots\right\} .
$$

If $\left(c_{n}\right) \in l^{\infty}$ then by (I) and (II) it is not hard to conclude that $\Sigma_{n} c_{n} f_{n} \in$ $F_{0}(G)$ and that $\left\|\Sigma_{n} c_{n} f_{n}+W_{0}(G)\right\|_{F_{0}(G) / w_{0}(G)}=\left\|\left(c_{n}\right)\right\|_{\infty}$. Therefore $\left(c_{n}\right)$ $\rightarrow \Sigma_{n} c_{n} f_{n}+W_{0}(G)$ is a linear isometry from $l^{\infty}$ into $F_{0}(G) / W_{0}(G)$ and we have proved the theorem if $G$ is $\sigma$-compact and unimodular.

(IV) If $G$ is not $\sigma$-compact but is unimodular, choose a $\sigma$-compact open subgroup $H$ of $G$ which contains a relatively noncompact portion of $X$. Construct $f_{n}$ on $H$ just as in (III). Consider $f_{n} \in C(G)$ by setting $f_{n} \equiv 0$ on $G \backslash H$. Then clearly $\left(c_{n}\right) \rightarrow \Sigma_{n} c_{n} f_{n}+W_{0}(G)$ is a linear isometry from $l^{\infty}$ into $F_{0}(G) / W_{0}(G)$.

(V) If $G$ is nonunimodular let $K=\{x \in G: \Delta(x)=1\}$. Then $G / K$ is isomorphic to a noncompact subgroup of the multiplicative group $\{x \in \mathbf{R}: x>0\}$. Denote the natural homomorphism from $G$ to $G / K$ by $\varphi$. Construct a sequence. of functions $f_{n}$ on $G / K$ as in (III). Then clearly $\left(c_{n}\right) \rightarrow \Sigma_{n} c_{n}\left(f_{n} \circ \varphi\right)+$ $W_{0}(G)$ is a linear isometry from $l^{\infty}$ into $F_{0}(G) / W_{0}(G)$.

REMARKS. (1) If $G$ is an arbitrary nonunimodular locally compact amenable group then $F_{0}(G) / W_{0}(G)$ contains a linear isometric copy of $l^{\infty}$ by part (V) of the above proof.

(2) Let $G$ be a non- $\sigma$-compact locally compact amenable group. Let $H$ be a $\sigma$-compact noncompact open subgroup of $G$. If $f \in U C(H), f^{\prime} \in U C(G)$ is defined by $f^{\prime} \equiv f$ on $H$ and $f^{\prime} \equiv 0$ on $G \backslash H$. The mapping from 
$U C(H) / W(H)$ to $F_{0}(G) / W_{0}(G)$ defined by sending $f+W(H)$ to $f^{\prime}+W_{0}(G)$ is a linear isometry. Since $U C(H) / W(H)$ is nonseparable $[9$, p. 62], so is $F_{0}(G) / W_{0}(G)$.

(3) If $G$ is a noncompact locally compact amenable group with property (E) then we can construct $\left(x_{i}\right),\left(y_{j}\right),\left(n_{k}\right)$ to satisfy (i)-(v) in the proof of Theorem 5.1. Keep the notation there and let

$$
f_{1}=\sum\left\{\frac{i-j}{i+j} l_{\left(x_{i} y_{j}\right)^{-1}} h: i \geqslant j\right\}, f_{2}=\sum\left\{\frac{i-j}{i+j} l_{\left(x_{i} y_{j}\right)^{-1}} h: i \leqslant j\right\} \text {. }
$$

Then $f_{1} \in F R_{0}(G) \backslash W_{0}(G)$ and $f_{2} \in F L_{0}(G) \backslash W_{0}(G)$.

A result parallel to the theorem on p. 62 of [9] is the following.

THEOREM 5.2. Let $G$ be a noncompact locally compact group with property (E). Then $U C(G) / W(G)$ contains a linear isometric copy of $l^{\infty}$.

Proof. Again we only have to consider the case that $G$ is $\sigma$-compact and unimodular. Let $X$ be a relatively noncompact $E$-set in $G$ and let $W$ be a compact neighborhood of $e$. Choose a compact symmetric neighborhood $V$ of $e$ such that $x V x^{-1} \subset W$ for $x \in X \cup X^{-1}$. Apply induction to obtain two sequences $\left(x_{i}\right),\left(y_{j}\right)$ of elements in $X$ such that $x_{i} y_{j} V \cap x_{i^{\prime}} y_{j^{\prime}} V=\varnothing$ if $(i, j) \neq\left(i^{\prime}, j^{\prime}\right)$. Construct a sequence of functions $\hat{j}_{n}$ from $\left(x_{i}\right),\left(y_{j}\right)$ as in part (III) of the proof of Theorem 5.1. Then ${ }^{\circ}\left(c_{n}\right) \rightarrow \Sigma_{n} c_{n} f_{n}+W(G)$ is a linear isometry of $l^{\infty}$ into $U C(G) / W(G)$.

REMARK. For convenience we shall say that a locally compact amenable group $G$ has property (*) if there exist two sequences $\left(X_{n}\right),\left(Y_{n}\right)$ of subsets of $G, X_{n}=\left\{x_{n i}\right\}_{i=1}^{\infty}, Y_{n}=\left\{y_{n j}\right\}_{j=1}^{\infty}$, and a sequence of functions $\left(f_{n}\right)$ on $G$ such that (i) for each $\left(c_{n}\right) \in l^{\infty}, \Sigma_{n} c_{n} f_{n} \in F_{0}(G)$, (ii) the supports of $f_{n}$ are mutually disjoint, (iii) $f_{n}\left(x_{n i} y_{n j}\right)=(i-j) /(i+j), n, i, j=1,2, \cdots$, and (iv) $\left\|f_{n}\right\|_{\infty}=1, n=1,2, \cdots$. It is clear that if $G$ has property $(*)$ then $F_{0}(G) / W_{0}(G)$ contains a linear isometric copy of $l^{\infty}$. In Theorem 5.1, we established that if $G$ is a locally compact amenable group with noncompact center then $G$ has property (*). It is also clear that if $G$ is a locally compact amenable group which has a continuous homomorphic image with property $(*)$ then $G$ also has property (*). In particular, we have the following.

COROLlaRY 5.3. Let $G$ be a noncompact locally compact nilpotent group. Then $F_{0}(G) / W_{0}(G)$ contains a linear isometric copy of $l^{\infty}$.

The proof of the above corollary is the same as that of Corollary 4.7. While Corollary 4.7 does not hold for the solvable connected Lie group in Example 2 of $\S 4$, Corollary 5.3 does have the following extension. 
THEOREM 5.4. If $G$ is an almost connected noncompact locally compact solvable group then $F_{0}(G) / W_{0}(G)$ contains a linear isometric copy of $l^{\infty}$.

Proof. Since $G$ is almost connected, there exists a compact normal subgroup $K$ such that $G / K$ is a Lie group with a finite number of components, cf. $[13$, p. 175]. We shall prove that the connected component of $G / K$ has property (*) which clearly implies that $G / K$ has property (*) and hence $G$ also has property (*). Therefore assume that $G$ itself is a noncompact connected solvable Lie group. By [12, Lemma 3.3] there exists a sequence of closed characteristic subgroups of $G$,

$$
G=G_{0} \supset G_{1} \supset \cdots \supset G_{n-1} \supset G_{n}=(e),
$$

such that $G_{i} / G_{i+1}$ is either a real vector group or a toroid, $i=0,1, \cdots, n-1$. We shall prove that $G$ has property (*) by induction on $n$. If $n=1$ then $G$ is a real vector group and hence has property $(*)$, by Theorem 5.1. Suppose that our assertion holds for $n-1(n>1)$. If $G / G_{n-1}$ is noncompact then by inductive assumption $G / G_{n-1}$ has property (*) and hence so does $G$. If $G / G_{n-1}$ is compact then it is $\mathrm{T}^{k}$ for some $k \geqslant 1$ and $G_{n-1}$ has to be $\mathbf{R}^{s}$ for some $s \geqslant 1$. Therefore $G$ is a split extension of $G_{n-1}, G=\mathbf{R}^{s} \times{ }_{\eta} \mathbf{T}^{k}$ where $\eta$ is a continuous homomorphism of $T^{k}$ into $A\left(R^{s}\right), \mathrm{cf}$. [12, Lemma 3.4]. It is well known that $A\left(\mathbf{R}^{\mathcal{S}}\right)=G L(s, \mathbf{R})$, cf. [11, p. 434]. Since $T^{k}$ is abelian, each irreducible (complex) representation of $\mathbf{T}^{k}$ is one dimensional. A simple complexification argument tells us that $\mathrm{R}^{s}$ can be written as $V_{1} \oplus \cdots \oplus V_{j}$ where each $V_{i}$ is either a one- or two-dimensional vector subspace of $\mathrm{R}^{s}$ and $\eta\left(\mathrm{T}^{k}\right) V_{i} \subset V_{i}$. Note that for each $i, V_{i} \times{ }_{\eta} \mathrm{T}^{k}$ is a homomorphic image of $G$. If one of the $V_{i}^{\prime}$ 's is one-dimensional then $\mathrm{R} \times \mathrm{T}^{k}$, being an abelian group, has property (*) and hence so does $G$. If each $V_{i}$ is two-dimensional over $\mathbf{R}$ then $\mathbf{R}^{2} \times{ }_{\eta} \mathbf{T}^{k}$ is a continuous homomorphic image of $G$. Therefore it is enough to prove that $G=\mathbf{R}^{2} \times_{\eta} \mathrm{T}^{k}$ has property (*). Let $I=\eta\left(\mathrm{T}^{k}\right)$. Assume that $I \neq(\delta)$ where $\delta$ denotes the identity matrix. Then $I$, being a connected compact abelian subgroup of $S L(2, \mathbf{R})$, is isomorphic to $\mathbf{T}$. In particular there exists an element $\iota$ of order 4 in $I$. Since $\iota^{4}-\delta=\left(\iota^{2}-\delta\right)\left(\iota^{2}+\delta\right)=0$,

$$
\iota^{2}=-1 \text {. }
$$

Let $M(2, \mathrm{R})$ be the algebra of $2 \times 2$ matrices over $\mathrm{R}$. Let $A_{1}$ and $A_{2}$ be the subalgebras of $M(2, \mathbf{R})$ generated by $\iota$ and $I$ respectively. Then both $A_{1}$ and $A_{2}$ are abelian, $A_{1} \subset A_{2}$ and $\operatorname{dim} A_{1}=2$. Since the dimension of each abelian subalgebra of $M(2, \mathrm{R})$ is less than or equal to two, $A_{1}=A_{2}$. In particular, if $\tau \in I$ then $\tau=x+y \iota$ for some $x, y \in \mathbf{R}, x^{2}+y^{2}=1$. Since $I$ is connected and $\left\{x+y\right.$ : $\left.x, y \in \mathbf{R}, x^{2}+y^{2}=1\right\}$ is isomorphic to $\mathrm{T}$, we have 


$$
I=\left\{x+y \imath: x, y \in \mathbf{R}, x^{2}+y^{2}=1\right\} .
$$

By (1) there exist $a, b \in \mathbf{R}, b \neq 0$ such that $\iota_{11}=-\iota_{22}=a, \iota_{12}=b, \iota_{21}=$ $-\left(1+a^{2}\right) / b$. Replacing $\iota$ by $-\imath$ if necessary, we shall assume that $b>0$. Clearly $\iota$ is similar to an orthogonal matrix $\omega$, i.e., there exists $\sigma \in G L(2, \mathbf{R})$ such that $\iota=\sigma^{-1} \omega \sigma$. Since $\omega^{2}=-1$ and $b>0, \omega_{11}=\omega_{22}=0, \omega_{12}=$ $-\omega_{21}=1$. So, by (2), $I=\left\{\sigma^{-1}(x+y \omega) \sigma: x, y \in \mathbf{R}, x^{2}+y^{2}=1\right\}$. Since the dual group of $\mathbf{T}^{k}$ is $\mathbf{Z}^{k}$, there exist $n_{1}, \cdots, n_{k} \in \mathbf{Z}$ such that if $\left(\exp \left(i \theta_{1}\right)\right.$, $\left.\cdots, \exp \left(i \theta_{k}\right)\right) \in \mathrm{T}^{k}$ then $\eta\left(\exp \left(i \theta_{1}\right), \cdots, \exp \left(i \theta_{k}\right)\right)=\sigma^{-1} \tau \sigma$ where $\tau_{11}=$ $\tau_{22}=\cos \left(n_{1} \theta_{1}+\cdots+n_{k} \theta_{k}\right)$ and $\tau_{12}=-\tau_{21}=\sin \left(n_{1} \theta_{1}+\cdots+n_{k} \theta_{k}\right)$. We shall identify $\mathbf{R}^{2}$ with $\mathbf{C}$ and hence multiplication in $G=\mathbf{C} \times \times_{\eta} \mathbf{T}^{k}$ is given by

$$
\begin{aligned}
\left(z ; \exp \left(i \theta_{1}\right), \cdots, \exp \left(i \theta_{k}\right)\right)\left(z^{\prime} ; \exp \left(i \theta_{1}^{\prime}\right), \cdots, \exp \left(i \theta_{k}^{\prime}\right)\right) \\
=\left(z+\sigma^{-1}\left(\exp \left(i\left(n_{1} \theta_{1}+\cdots+n_{k} \theta_{k}\right)\right)\left(\sigma z^{\prime}\right)\right) ;\right. \\
\quad \exp \left(i\left(\theta_{1}+\theta_{1}^{\prime}\right), \cdots, \exp \left(i\left(\theta_{k}+\theta_{k}^{\prime}\right)\right)\right) .
\end{aligned}
$$

Note that if $(z, t),\left(z^{\prime}, t^{\prime}\right) \in G, t=\left(\exp \left(i \theta_{1}\right), \cdots, \exp \left(i \theta_{k}\right)\right)$, then

$$
\begin{aligned}
|| \sigma\left(z+\eta(t) z^{\prime}\right)|-| \sigma z|| & \leqslant \mid \sigma z+\exp \left(i\left(n_{1} \theta_{1}+\cdots+n_{k} \theta_{k}\right)\right) \sigma z^{\prime} \\
& -\exp \left(i\left(n_{1} \theta_{1}+\cdots+n_{k} \theta_{k}\right)\right) \sigma z^{\prime} \mid \\
& =|\sigma z|,
\end{aligned}
$$

and

$$
|| \sigma\left(z^{\prime}+\eta\left(t^{\prime}\right) z\right)|-| \sigma z^{\prime}|| \leqslant|\sigma z| .
$$

Therefore, we have

(3) If $g \in U C(\mathbf{R})$ and if $f$ is defined by $f(z, t)=g(|\sigma z|),(z, t) \in G$, then $f \in U C(G)$.

Choose three strictly increasing sequences of positive integers $\left(a_{i}\right),\left(b_{j}\right)$, ( $n_{s}$ ) such that (a) $n_{s+1} \geqslant a_{i}+b_{j} \geqslant n_{s}$, (b) $a_{i}-a_{i^{\prime}} \geqslant n_{i-1}, b_{j}-b_{j^{\prime}} \geqslant n_{j-1}$ if $i>i^{\prime}, j>j^{\prime}$, (c) $n_{s} \geqslant 2^{s}$. Write $\left\{a_{i}\right\}=\left\{a_{n i}: n, i=1,2, \cdots\right\},\left\{b_{j}\right\}=\left\{b_{n j}\right.$ : $n, j=1,2, \cdots\}$ such that $a_{n i} \neq a_{n^{\prime} i^{\prime}}$ if $(n, i) \neq\left(n^{\prime}, i^{\prime}\right)$ and $b_{n j} \neq b_{n^{\prime} j^{\prime}}$ if $(n, j) \neq\left(n^{\prime}, j^{\prime}\right)$. Let $h$ be a continuous function as $\mathbf{R}$ with $h(0)=1,0 \leqslant h$ $\leqslant 1, h \equiv 0$ outside of the open interval $(-1 / 2,1 / 2)$. Let

$$
g_{n}=\sum\left\{\frac{i-j}{i+j} l_{\left(-a_{n i}-b_{n j}\right)} h: i, j=1,2, \cdots\right\} \text { and } f_{n}(z, t)=g_{n}(|\sigma z|) \text {. }
$$

Then, by (3), for each $\left(c_{n}\right) \in l^{\infty}, \Sigma_{n} c_{n} f_{n} \in U C(G)$. It is easy to see that $G$ is unimodular and that the Haar measure for $G$ is the product of the Haar measure 
for $\mathbf{C}$ and the Haar measure for $\mathrm{T}^{k}$ (cf. $[11, \mathrm{p} .210]$ ). It is also easily checked that $W_{n}=\{z \in \mathbf{C}:|\sigma z| \leqslant n\} \times \mathrm{T}^{k}, n=1,2, \cdots$, is an $F$-sequence for $G$ and that each $W_{n}$ is symmetric. We claim that for each $\left(c_{n}\right) \in l^{\infty}, \Sigma_{n} c_{n} f_{n} \in F_{0}(G)$. The proof of this claim is not very short but is quite routine. We like to leave the details to the reader. Since $f_{n}\left(\sigma^{-1}\left(a_{n i}+b_{n j}\right), 1\right)=g\left(a_{n i}+b_{n j}\right)=(i-j) /(i+j)$ we have finally completed the proof of the fact that $G$ has property $(*)$.

REMARK. Let $G$ be a noncompact locally compact group which is either nilpotent or is almost connected and solvable. Then, by Corollary 5.3 and Theorem 5.4, $F_{0}(G) \oplus A(G) / W(G)$ contains a linear isometric copy of $l^{\infty}$. It implies that $F(G) / W(G)$ contains a linear isometric copy of $l^{\infty}$. It also implies that $W(G)$ is not a maximal subalgebra of $F(G)$. We do not know whether $F_{0}(G) \oplus A(G)$ is a maximal subalgebra of $F(G)$.

\section{REFERENCES}

1. R. B. Burckel, Weakly almost periodic functions on semigroups, Gordon and Breach, New York, 1970. MR 41 \#8562.

2. C. Chou, On the size of the set of left invariant means on a semigroup, Proc. Amer. Math. Soc. 23 (1969), 199-205. MR 40 \#710.

3. - On topologically invariant means on a locally compact group, Trans. Amer. Math. Soc. 151 (1970), 443-456. MR $42 \# 4675$.

4. C. Chou and J. P. Duran, Multipliers for the space of almost-convergent functions on a semigroup, Proc. Amer. Math. Soc., 39 (1973), 125-128.

5. M. M. Day, Semigroups and amenability, (Proc. Sympos. Semigroups, Wayne State Univ., Detroit, Michigan, 1968), Academic Press, New York, 1969, pp. 5-53. MR 42 \#11.

6. K. deLeeuw and I. Glicksberg, The decomposition of certain group representations, Analyse Math. 15 (1965), 135-192. MR 32 \#211.

7. N. Dunford and J. T. Schwartz, Linear operators. I: General theory, Pure and Appl. Math., vol. 7, Interscience, New York, 1958. MR 22 \#302.

8. W. F. Eberlein, Abstract ergodic theorems and weak almost periodic functions,

Trans. Amer. Math. Soc. 67 (1949), 217-240. MR 12, 112.

9. E. E. Granirer, Exposed points of convex sets and weak sequential convergence, Mem. Amer. Math. Soc. No. 123, 1972.

10. F. P. Greenleaf, Invariant means on topological groups and their applications, Van Nostrand-Reinhold Math. Studies, no. 16, Van Nostrand-Reinhold, New York, 1969. MR 40 \#4776.

11. E. Hewitt and K. A. Ross, Abstract harmonic analysis. Vol. 1: Structure of topological groups. Integration theory, group representations, Die Grundlehren der math. Wissenschaften, Band 115, Academic Press, New York; Springer-Verlag, Berlin, 1963. MR 28 \#158.

12. K. Iwasawa, On some types of topological groups, Ann. of Math. (2) 50 (1949), 507-558. MR 10, 679.

13. D. Montgomery and L. Zippin, Topological transformation groups, Interscience, New York, 1955. MR 17, 383.

14. W. Rudin, Weak almost periodic functions and Fourier-Stielties transforms, Duke Math. J. 26 (1959), 215-220. MR 21 \#1492.

15. J. C. S. Wong, Topologically stationary locally compact groups and amenability, Trans. Amer. Math. Soc. 144 (1969), 351-363. MR 40 \#2781. 